\title{
Températures de surface et mesures mobiles confrontées aux zones climatiques locales : exemples des agglomérations de Tokyo et de Lyon
}

\author{
Florent Renard * et Lucille Alonso \\ Université Jean Moulin Lyon 3, UMR 5600 CNRS Environnement Ville Société, Lyon, France
}

\begin{abstract}
Résumé - Les zones climatiques locales (ZCL) sont de plus en plus utilisées dans toutes les problématiques liées au changement climatique en milieu urbain, notamment pour les opérations d'aménagement ou de requalification portant sur l'îlot de chaleur, les microclimats et le confort thermique. Fondées sur des critères a priori, ces dernières nécessitent d'être confrontées à la réalité du terrain afin de s'assurer de leur validité avant toute utilisation. C'est l'objet de cette étude portant sur les agglomérations témoins de Lyon et de Tokyo, où ces zones climatiques n'ont pour le moment pas été réellement utilisées. L'analyse proposée confronte ce découpage thermo-morphologique aux températures de surface provenant des mesures de Landsat de 2000 à 2019 (single channel algorithm) et de mesures mobiles de la température de l'air, effectuées au sein des agglomérations en été. Les ZCL sont extraites par photo-interprétation pour Lyon et par classification supervisée pour Tokyo. Les températures sont étudiées en fonction des ZCL à l'aide du test statistique de Kruskal-Wallis suivi de la procédure de comparaison multiple par paires Steel-Dwass-Critchlow-Fligner. Les résultats indiquent des différences significatives entre les ZCL pour les températures de l'air et de surface et confirment ainsi l'intérêt de leur utilisation. Les ZCL les plus fraîches sont celles qui présentent une couverture végétalisée. Plus précisément, on remarque un gradient thermique décroissant avec la quantité de biomasse disponible au sein des ZCL mais également avec la hauteur des bâtiments en raison de l'effet d'ombrage.
\end{abstract}

Mots-clés : zones climatiques locales / température de l'air / température de surface / morphologie urbaine / Landsat / gradients thermiques / classification random forest.

\begin{abstract}
Surface temperatures and mobile measurements compared to local climate zones: examples of the Tokyo and Lyon conurbations. Local Climate Zones (LCZs) are increasingly used in all issues related to climate change in urban areas, particularly for development or requalification operations involving heat islands, micro-climates and thermal comfort. Based on a predefined set of criteria, these require a comparison with the reality on the ground in order to ensure their validity before any use is made of them. This is the purpose of this study on the reference agglomerations of Lyon and Tokyo, where these climatic zones have not yet been really used. The proposed analysis compares this thermo-morphological subdivision with surface temperatures from Landsat measurements from 2000 to 2019 (single channel algorithm) and mobile air temperature measurements taken within the agglomerations in summer. The LCZs are extracted by photo-interpretation for Lyon and by supervised classification for Tokyo. Temperatures are studied as a function of LCZs using the statistical KruskalWallis test followed by the Steel-Dwass-Critchlow-Fligner multiple pair comparison procedure. The results indicate significant differences between LCZs for air and surface temperatures and thus confirm the value of their use. The coolest LCZs are those with a vegetated cover. More specifically, a decreasing thermal gradient can be observed with the amount of biomass available within the LCZs but also with the height of the buildings due to the shading effect.
\end{abstract}

Keywords: local climate zones / air temperature / surface temperature / urban morphology / Landsat / thermal gradients / classification random forest.

\footnotetext{
* $\overline{\text { Auteur de correspondance }}$ : florent.renard@univ-lyon3.fr
} 


\section{Introduction}

Le changement climatique actuel est susceptible de renforcer l'intensité et la fréquence des événements thermiques extrêmes (Pachauri et Meyer, 2014). C'est par exemple le cas des vagues de chaleur dont la sévérité augmente sur l'Europe, notamment depuis les dernières décennies (Meehl et Tebaldi, 2004 ; Fink et al., 2006 ; Della Marta et al., 2007 ; IPCC, 2013). Les milieux urbains sont encore plus sensibles à ces élévations de température en raison de l'effet d'îlot de chaleur urbain ou ICU (Weston, 1987; Voogt et Oke, 2003).

L'augmentation de chaleur en milieu urbain est l'effet conjugué de paramètres surfaciques (faible évapotranspiration et évaporation liées au remplacement du couvert végétal, des zones humides et des surfaces en eau par des surfaces artificialisées), des propriétés physiques du bâti (les matériaux à faible albédo et à forte inertie thermique absorbent et stockent beaucoup plus la chaleur que le couvert végétal), de paramètres morphologiques (par exemple, la rugosité urbaine diminue la vitesse des vents, un faible sky view factor entraine un piégeage du rayonnement et limite le dégagement de chaleur la nuit) et de paramètres anthropiques (chaleur produite par l'industrie, le chauffage, le transport, la climatisation, etc. (de Munck et al., 2018). De ce fait, il est commun de trouver des différences de températures en faveur du milieu urbain de 5 à $10^{\circ} \mathrm{C}$ environ par rapport aux zones rurales en périphéries, lors de conditions climatiques favorables, notamment un ciel clair et une absence de vent (Azevedo et al., 2016).

Les métropoles de Lyon et de Tokyo, territoires d'étude de ce travail, ne font pas exception à la règle. Des solutions doivent être trouvées via les politiques d'aménagement urbain en implémentant des stratégies d'adaptation durable, comme celles recommandées par la plateforme européenne d'adaptation climatique ou l'Ademe, notamment en accroissant les espaces verts, ombragés et humides dans les secteurs les plus favorables à la surchauffe urbaine (Lin et al., 2017) afin d'améliorer le confort thermique des habitants (Qaid et al., 2013, 2018 ; Desai et Dhorde, 2018).
Dans une optique d'amélioration des conditions de vie des citadins, il convient ainsi de limiter au maximum cet effet d'ICU. Cela passe obligatoirement dans un premier temps par une connaissance fine du phénomène climatique, c'està-dire par une appréciation physique des gradients thermiques et des microclimats urbains. L'amélioration du bien-être des citadins passe également par une connaissance préalable des formes d'habitat afin d'y appliquer des stratégies de renouvellement urbain favorisant le confort thermique. En effet, les diversités de morphologie urbaine peuvent considérablement modifier les microclimats et le confort thermique en conséquence (Demuzere et al., 2019).

A cette fin, la classification la plus utilisée au niveau mondial est celle reposant sur les zones climatiques locales (ZCL) de Stewart et Oke (2012). Ce concept de classification propose ainsi d'utiliser une méthodologie commune pour définir les caractéristiques physiques des villes à l'intention des urbanistes, architectes et climatologues urbains notamment. Par conséquent, cette terminologie est transférable dans toutes les zones géographiques et culturelles. Les 17 zones principales sont établies en fonction des caractéristiques de la structure de surface.

Cette classification n'a cependant jamais été utilisée ni sur Lyon, ni sur Tokyo, à notre connaissance. Ces deux territoires d'études ont ainsi été choisis pour cette raison principale, mais aussi pour leurs climats ainsi que leurs morphologies urbaines différentes. Ils s'inscrivent en outre dans les échanges existants depuis longtemps entre ces deux villes dans le cadre de l'Ecole Urbaine de Lyon qui a facilité cette analyse en permettant des études de terrain.

Il convient donc dans un premier temps de réaliser ces classifications d'occupation du sol puis de les mettre à l'épreuve des données thermiques afin de s'assurer de leurs cohérences morphoclimatiques, avant leurs utilisations potentielles dans des projets de réaménagement ou dans des modèles de simulations climatiques locales et régionales. Cela peut être effectué par le biais d'un réseau de mesures fixes suffisamment dense s'il existe (Richard et al., 2018 ; Foissard et al., 2019), 
par des mesures mobiles (Leconte et al., 2017) ou par des données thermiques satellitaires (Alonso et Renard, 2019). Les réseaux météorologiques de Lyon et de Tokyo n'étant pas assez développés, les deux derniers protocoles seront utilisés dans cette étude.

De ce fait, la deuxième partie de ce texte est dédiée à la présentation des deux territoires d'étude, Lyon (France) et Tokyo (Japon), et des données et méthodes utilisées pour obtenir les ZCL. Les données de température de l'air et de surface utilisées pour confronter les ZCL sont également explicitées. La troisième partie se concentre sur les résultats de l'évaluation des ZCL par rapport aux données thermiques. Enfin, la dernière section est consacrée à la discussion de ces résultats et aux principales réflexions, ainsi qu'aux forces et aux limites des méthodologies des classifications, accompagnées des futures perspectives de recherche.

\section{Détermination des zones climatiques locales et mesure des températures}

\subsection{Zones climatiques locales : photo- interprétation et classification supervisée}

La classification des ZCL comprend dix catégories urbaines (ZCL 1 à 10) et sept avec des couverts naturels (ZCL A à G), à l'exception d'une catégorie qui inclut aussi des surfaces pavées (E). Chacune des ZCL est conçue pour potentiellement avoir son propre microclimat qui diffèrerait de ses voisins et à une superficie pouvant aller de 10000 $\mathrm{m}^{2}$ à quelques kilomètres carrés. L'élaboration des ZCL est fondée sur l'homogénéité et la cohérence des propriétés géomorphologiques du secteur (le pourcentage de surface imperméabilisé, le sky view factor, etc.), environnementales (la présence et la nature du couvert végétal), métabolique (production de chaleur anthropique) et thermique et radiative (admittance et albédo de la surface) (Bechtel et al., 2015, 2017 ; Demuzere et al., 2019 ; Verdonck et $a l ., 2019)$. Cette classification a été utilisée dans de multiples études microclimatiques de par le monde et a prouvé sa cohérence morpho-climatique (Alexander et al., 2015 ; Brousse et al., 2016 ;
Richard et al., 2018 ; Molnár et al., 2019).

Les ZCL de l'agglomération lyonnaise sont obtenues par photo-interprétation d'imagerie aérienne. L'interprétation est réalisée par les auteurs de cette étude, pouvant être qualifiés d'opérateurs qualifiés possédant une solide connaissance du territoire. La photo-orthographie utilisée date d'avril 2018 et possède une résolution de $8 \mathrm{~cm}$, disponible sur la plateforme data du Grand Lyon.

Cette photo-interprétation peut également être confrontée aux prises de vue de Google Maps et de Google Earth, complétée par des vérifications sur le terrain. De cette manière, 4922 polygones ont été obtenus sur les $491 \mathrm{~km}^{2}$ de l'agglomération comptant 1,4 millions d'habitants (figure 1).

En revanche, une photo-interprétation n'était pas envisageable pour l'agglomération de Tokyo, du fait de son étendue sur $12733 \mathrm{~km}^{2}$ et du travail analytique nécessaire en conséquence. Ainsi, une classification supervisée orientée objet selon l'algorithme Random forests a été réalisée afin d'identifier les ZCL. Il s'agit d'une classification effectuée sur des zones de pixels (et non sur un seul pixel comme pour les classifications orientées pixel), regroupés selon une segmentation (Jones et Reinke, 2009). Ce processus prend en compte les caractéristiques de couleurs et de formes lors du regroupement des pixels en objets. Les objets produits par la segmentation concordent mieux aux caractéristiques du terrain et fournissent des résultats de classification plus spécifiques (Jones et Reinke, 2009).

Cette classification Random forests a été utilisée préférentiellement à d'autres méthodes non supervisées (ISO Cluster) ou supervisées (Maximum Likelihood ou Support Vector Machine), en raison de sa performance (Breiman, 2001 ; Grömping, 2009 ; Breiman et al., 2017). Celle classification a été réalisée avec les bandes visible, proche infrarouge et thermique de données de Landsat (USGS, résolution de $30 \mathrm{~m}$ ) et Sentinel (ESA - Copernicus, 10 m) d'avril 2017. Le secteur d'étude correspond aux zones recoupant les dalles des satellites des districts de Tokyo, Saitama, Kanagawa et Chiba (figure 2). 


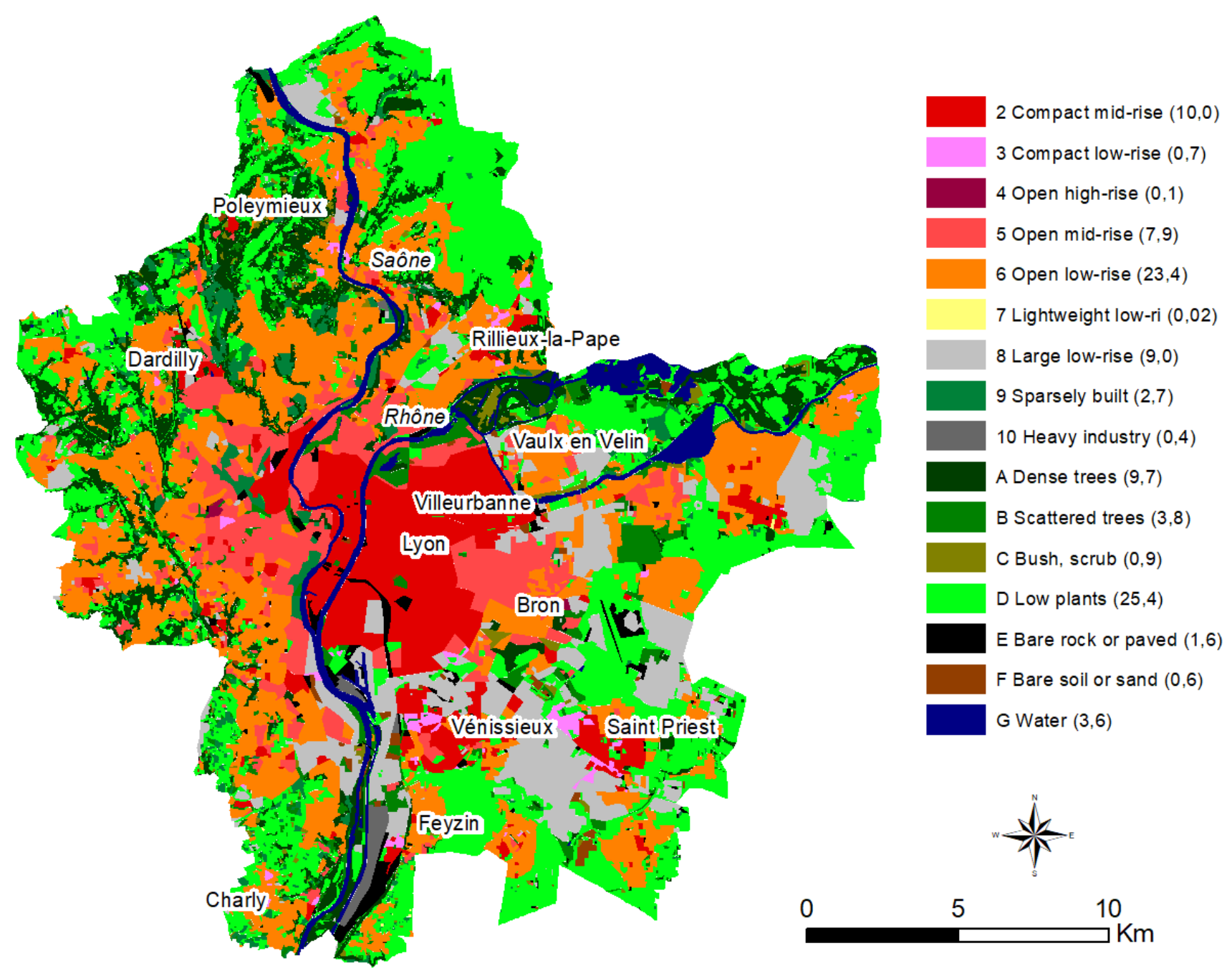

Figure 1. Les 17 Zones Climatiques Locales (ZCL) de la métropole de Lyon et leurs superficies (\%) (photo-interprétation à partir de l'orthophotographie d'avril 2018). The 17 Local Climate Zones (LCZ) of the metropolis of Lyon and their areas (\%) (photo-interpretation based on the orthophotography of April 2018).

La matrice de confusion de la classification Random forests sur l'agglomération de Tokyo donne des résultats très satisfaisants (tableau 1). Les précisions de l'utilisateur (faux positifs - erreurs de commission ou erreur de type 1) ne sont jamais inférieures à 0,80 et atteignent même 0,89 pour la classe large low-rise. Les précisions des producteurs (faux négatifs - erreurs d'omission ou erreur de type 2) sont également élevées. La valeur la plus faible est 0,82 (bare rock or paved) et la plus forte est toujours pour la classe large low-rise avec 0,93. Enfin, le coefficient Kappa de Cohen d'accord global de 0,85 indique ainsi que les résultats de la classification sont très bons (Landis et Koch, 1977). Cette classification a en outre été par la suite validée et amendée à l'aide d'informations issues de Google
Maps et de Google Earth, grâce à l'expertise de collègues japonais et d'observations sur le terrain.

\subsection{Températures de surface : utilisation du single channel algorithm}

Les températures de surface ont été obtenues à l'aide du single channel algorithm (Sobrino et al., 2004 ; Renard et al., 2019). Toutes les images de Landsat présentant une couverture nuageuse inférieure à $10 \%$ ont été traitées de 2000 à mi-2019 : 62 pour Lyon et 59 pour Tokyo (tableaux 2 et 3 ). De plus, seuls les pixels présentant une qualité suffisante selon la band quality assessment ont été conservés, c'est-à-dire les pixels qualifiés de Low Cloud Confidence, Low Cloud Shadow Confidence, Low Snow /Ice Confidence (figure 3). 


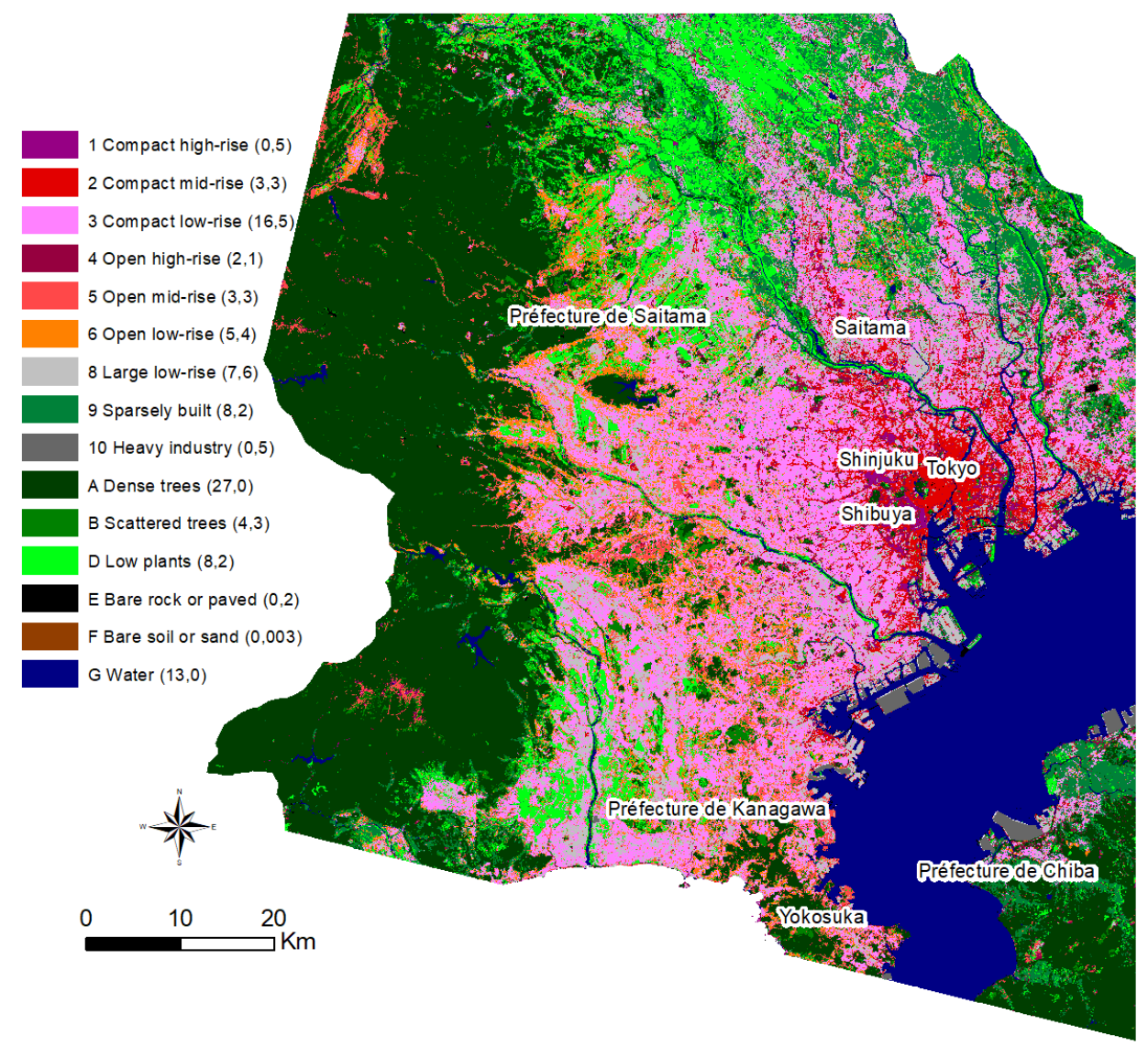

Figure 2. Les 16 Zones Climatiques Locales de la mégapole de Tokyo calculées à l'aide d'une classification Random forests à partir de données Landsat et Sentinel d'avril 2017 et leurs superficies (\%). The 16 Local Climate Zones of the Tokyo metropolis calculated using a Random forests classification based on Landsat and Sentinel data from April 2017 and their superficies (\%).

Tableau 1. Matrice de confusion de la classification Random forests sur l'agglomération de Tokyo entre les ZCL classées et les données de référence par photo-interprétation. Confusion matrix of the Random forests classification over the Tokyo metropolitan area between classified LCZs and reference data by photo-interpretation.

\begin{tabular}{|c|c|c|c|c|c|c|c|c|c|c|c|c|c|c|c|}
\hline & 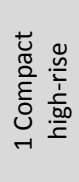 & 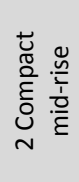 & 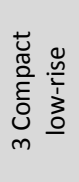 & 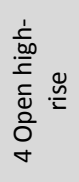 & 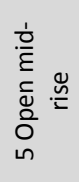 & 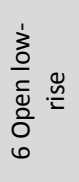 & 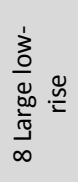 & 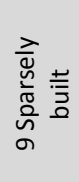 & 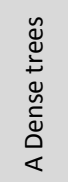 & 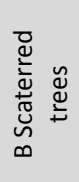 & $\begin{array}{l}\frac{n}{0} \\
\stackrel{0}{0} \\
\frac{0}{2} \\
3 \\
0 \\
0 \\
0\end{array}$ & 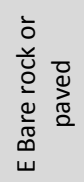 & 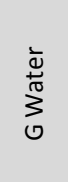 & $\begin{array}{l}\bar{\pi} \\
\stackrel{0}{0}\end{array}$ & 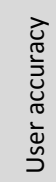 \\
\hline 1 Compact high-rise & 172 & 9 & 8 & 3 & 5 & 0 & 1 & 1 & 0 & 0 & 3 & 5 & 7 & 214 & 0,80 \\
\hline 2 Compact mid-rise & 8 & 175 & 3 & 2 & 4 & 3 & 0 & 0 & 2 & 0 & 0 & 2 & 5 & 204 & 0,86 \\
\hline 3 Compact low-rise & 6 & 5 & 179 & 4 & 6 & 8 & 3 & 2 & 0 & 3 & 2 & 1 & 4 & 223 & 0,80 \\
\hline 4 Open high-rise & 2 & 1 & 2 & 169 & 5 & 4 & 2 & 0 & 5 & 4 & 0 & 0 & 0 & 194 & 0,87 \\
\hline 5 Open mid-rise & 4 & 3 & 1 & 2 & 180 & 8 & 1 & 2 & 3 & 2 & 1 & 0 & 0 & 207 & 0,87 \\
\hline 6 Open low-rise & 3 & 4 & 2 & 5 & 3 & 173 & 3 & 1 & 2 & 1 & 2 & 3 & 1 & 203 & 0,85 \\
\hline 8 Large low-rise & 0 & 2 & 1 & 4 & 2 & 2 & 175 & 0 & 0 & 0 & 0 & 8 & 2 & 196 & 0,89 \\
\hline 9 Sparsely built & 0 & 3 & 1 & 3 & 3 & 1 & 0 & 178 & 8 & 9 & 5 & 3 & 0 & 214 & 0,83 \\
\hline A Dense trees & 0 & 0 & 0 & 4 & 5 & 1 & 0 & 8 & 172 & 11 & 5 & 1 & 3 & 210 & 0,82 \\
\hline B Scaterred trees & 1 & 0 & 1 & 3 & 2 & 0 & 1 & 7 & 3 & 169 & 6 & 2 & 1 & 196 & 0,86 \\
\hline D Low plants & 0 & 0 & 0 & 1 & 0 & 3 & 1 & 6 & 3 & 4 & 181 & 6 & 4 & 209 & 0,87 \\
\hline E Bare rock or paved & 3 & 0 & 1 & 0 & 4 & 2 & 1 & 3 & 0 & 2 & 7 & 169 & 3 & 195 & 0,87 \\
\hline G Water & 5 & 0 & 2 & 4 & 1 & 3 & 0 & 2 & 0 & 1 & 6 & 7 & 181 & 212 & 0,85 \\
\hline Total & 204 & 202 & 201 & 204 & 220 & 208 & 188 & 210 & 198 & 206 & 218 & 207 & 211 & 2677 & \\
\hline Producer accuracy & 0,84 & 0,86 & 0,89 & 0,82 & 0,81 & 0,83 & 0,93 & 0,84 & 0,86 & 0,82 & 0,83 & 0,81 & 0,84 & & 0,85 \\
\hline
\end{tabular}


Tableau 2. Journées de passage des satellites Landsat $(5,7$ ou 8$)$ utilisées pour la sélection des images à l'échelle de l'agglomération de Lyon. Days of passage of Landsat satellites $(5,7$ or 8$)$ used for the selection of images at the scale of the Lyon metropolitan area.

\begin{tabular}{|c|c|c|c|c|c|c|c|c|c|}
\hline 2000 & 10-août & 11-sept. & 22-oct. & & & & & & \\
\hline 2001 & 31-mars & 26-juin & 21-juil. & 29-juil. & 13-août & 16-oct. & 25-oct. & & \\
\hline 2002 & 3-avr. & 14-juin & 22-juin & 16-août & & & & & \\
\hline 2003 & 10-juil. & 19-juil. & 4-août & 11-août & 27-août & 21-sept. & & & \\
\hline 2004 & 25-mai & 10-juin & 26-juin & 21-juil. & & & & & \\
\hline 2005 & 22-juin & & & & & & & & \\
\hline 2006 & 9-juin & 29-sept. & & & & & & & \\
\hline 2011 & 11-avr. & & & & & & & & \\
\hline 2013 & 25-avr. & 14-juil. & 21-juil. & 15-août & 22-août & 31-août & 22-sept. & & \\
\hline 2014 & 12-avr. & 5-mai & 1-juil. & 17-juil. & 26-sept. & 31-déc. & & & \\
\hline 2015 & 6-avr. & 4-juil. & 11-juil. & 5-août & 12-août & 21-août & 28-août & 29-sept. & 9-nov. \\
\hline 2016 & 13-févr. & & & & & & & & \\
\hline 2017 & 20-avr. & 22-mai & 29-mai & & & & & & \\
\hline 2018 & 24-janv. & 25-mai & 26-juin & 3-juil. & 12-juil. & 16-oct. & & & \\
\hline 2019 & 4-janv. & 21-févr. & & & & & & & \\
\hline
\end{tabular}

Tableau 3. Journées de passage des satellites Landsat $(5,7$ ou 8$)$ utilisées pour la sélection des images à l'échelle de l'agglomération de Tokyo. Days of passage of Landsat satellites $(5,7$ or 8$)$ used for the selection of images at the scale of the Tokyo metropolitan area.

\begin{tabular}{|c|c|c|c|c|c|}
\hline 2000 & 29-mars & 21-sept. & 7-oct. & 24-nov. & 2-déc. \\
\hline 2001 & 4-juin & 24-sept. & 11-nov. & 27-nov. & \\
\hline 2002 & 14-janv. & 19-mars & 7-juin & 13-oct. & 14-nov. \\
\hline 2003 & 17-janv. & & & & \\
\hline 2004 & 28-janv. & 13-févr. & 4-juin & 27-nov. & 13-déc. \\
\hline 2005 & 19-mars & 27-mars & & & \\
\hline 2006 & 16-oct. & & & & \\
\hline 2007 & 5-févr. & 21-févr. & 10-avr. & 12-mai & \\
\hline 2008 & 11-mars & 21-oct. & 22-nov. & & \\
\hline 2009 & 25-janv. & 10-févr. & 1-mai & & \\
\hline 2010 & 23-juil. & 11-oct. & 28-nov. & & \\
\hline 2011 & 5-avr. & 10-juil. & & & \\
\hline 2013 & 17-sept. & 20-nov. & & & \\
\hline 2014 & 7-janv. & 23-janv. & 28-mars & 31-mai & 9-déc. \\
\hline 2015 & 31-mars & 2-mai & 9-oct. & 25-oct. & \\
\hline 2016 & 17-mars & 27-oct. & & & \\
\hline 2017 & 31-janv. & 16-févr. & 20-mars & & \\
\hline 2018 & 2-janv. & 1-oct. & 2-nov. & & \\
\hline 2019 & 5-janv. & 21-janv. & & & \\
\hline
\end{tabular}

\subsection{Températures de l’air : des mesures itinérantes}

Après avoir testé les mesures en automobile, ces dernières ont été rejetées car elles présentaient trop de biais méthodologiques et de limites pratiques: problèmes de congestion du trafic, impossibilité de mesurer les zones piétonnes, mesures sur le réseau viaire uniquement, coût, temps, pollution, proximité avec la chaleur anthropique des moteurs à explosion, etc. Ainsi, sur Lyon, des mesures de température de l'air à vélo à vitesse modérée $(<10$ $\mathrm{km} / \mathrm{h}$ ) ont plutôt été effectuées. Après comparaison, elles présentent des résultats équivalents aux mesures pédestres mais permettent de couvrir un plus grand territoire sur un même intervalle de temps. Elles provoquent en outre moins de fatigue au personnel sur le terrain.

Sur Tokyo, des mesures pédestres ont été réalisées car il nous a semblé trop dangereux de 
pratiquer le vélo dans cette agglomération où ce mode de déplacement n'est pas fortement répandu, notamment en raison de l'efficacité du réseau de métro. En outre, les pistes cyclables ne sont pas nombreuses comme à Lyon et il est obligatoire de rouler sur le trottoir.

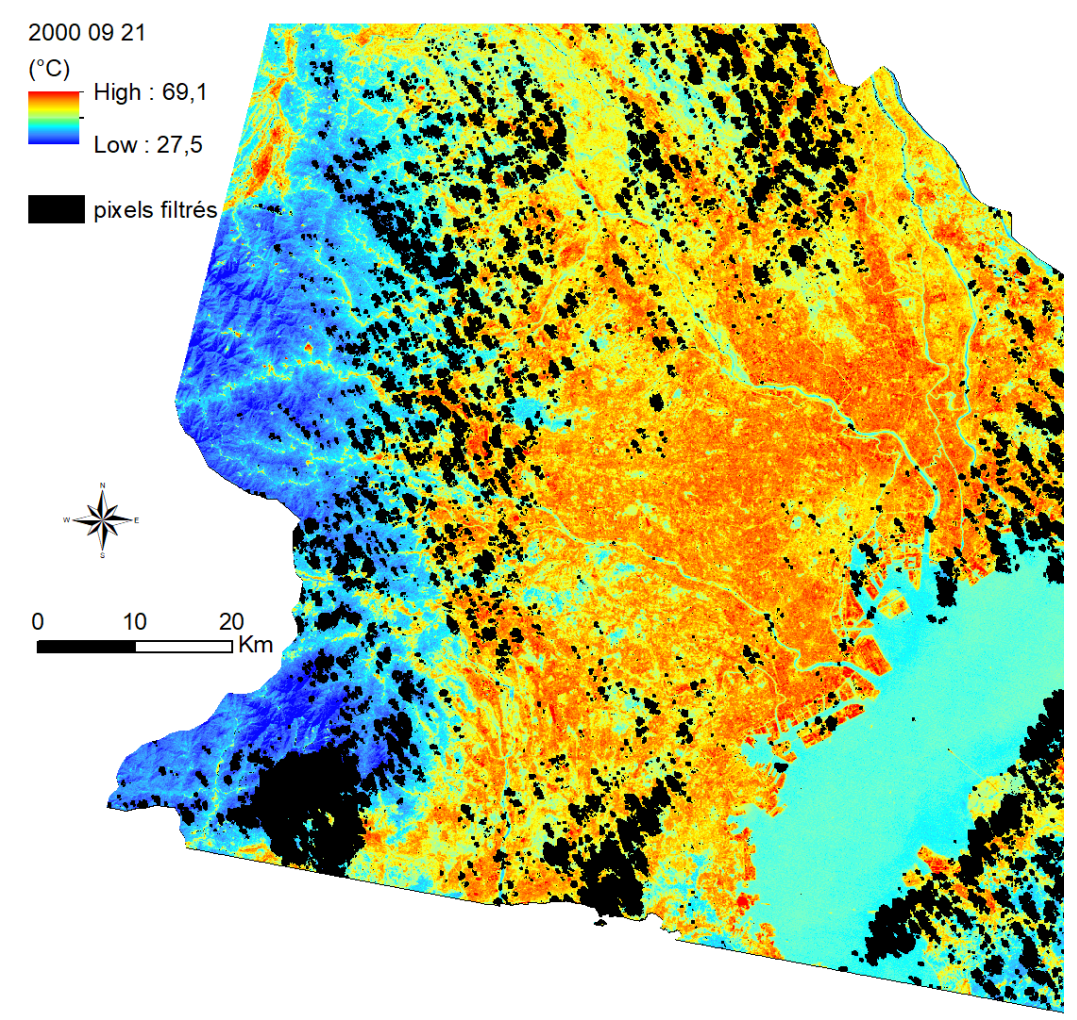

Figure 3. Exemple des températures de surface du 21 septembre 2000 estimées par imagerie Landsat sur Tokyo, sans (à gauche) et avec filtrage (à droite) des pixels de moindre qualité (325 188 pixels sur 1314917 ont été filtrés, soit 24,7 $\%$ de la superficie étudiée). Example of surface temperatures on September 21, 2000 estimated by Landsat imagery over Tokyo, without (left) and with filtering (right) of lower quality pixels. 325188 pixels by 1314917 were filtered, i.e. $24.7 \%$ of the area studied.

Les trajets sur les deux villes ont été choisis en fonction d'un ensemble de paramètres qui visaient à conjuguer au mieux durée du parcours, diversité morphologique, fréquentation des quartiers, marchabilité et cyclabilité.

Un enregistreur continu Log32 a été utilisé, sous un abri anti-radiation normalisé Davis Instruments - 7714. La localisation est assurée par un GPS Garmin 64s. De nombreuses campagnes de mesure ont été réalisées sur les deux sites durant les mois d'été 2016 à 2019. Dans le cadre de cette étude, seules les mesures de l'après-midi sont exploitées, soit 18 campagnes de mesure sur Lyon (tableau 4) et 12 sur Tokyo (tableau 5), avec des conditions de mesure optimales pour l'étude des gradients thermiques, i.e. par ciel dégagé et absence de vent. Les mesures ont été effectuées essentiellement en début d'après-midi afin de trouver un compromis entre le maximum thermique et le passage héliosynchrone de Landsat aux alentours de 12h30 sur Lyon. Les mesures effectuées sur des ponts sont considérées comme des mesures ayant été faites dans la catégorie de ZCL eau.

\subsection{Tests statistiques: une procédure de comparaison multiple}

Les données de températures de l'air et de surface associées à chaque échantillon de ZCL ne suivent pas une distribution normale (Shapiro et Wilk, 1965) et les variances des échantillons ne sont pas homogènes (Box, 1953) : il n'est donc pas conseillé d'utiliser une ANOVA mais de se tourner vers le test non-paramétrique de Kruskal-Wallis suivi d'une procédure de comparaison multiple par paires, afin d'étudier les variabilités inter- et intraclasses. Plusieurs méthodes existent comme celles 
de Dunn (Dunn, 1964), Conover et Iman (Conover, 1998) ou encore de Steel-Dwass-Critchlow-Fligner (Fligner, 1984). Notre choix s'est porté sur la dernière, comme recommandé par Hollander et
Wolfe (1999). Le test de Steel-Dwass-CritchlowFligner peut aussi être vu comme une alternative complète au test de Kruskal-Wallis.

Tableau 4. Mesures micro-météorologiques mobiles réalisées en été entre 2016 et 2019 utilisées pour l'agglomération de Lyon. Mobile micro-meteorological measurements carried out in summer between 2016 and 2019 used for the Lyon metropolitan area.

\begin{tabular}{|c|c|c|c|c|c|c|c|c|c|c|c|c|}
\hline \multirow[b]{2}{*}{ Date } & \multicolumn{2}{|c|}{ Heure locale } & \multicolumn{2}{|c|}{$\begin{array}{c}\text { Pression } \\
\text { barométrique } \\
(\mathrm{hPa})\end{array}$} & \multicolumn{2}{|c|}{$\begin{array}{l}\text { Précipitation } \\
\text { (mm) }\end{array}$} & \multicolumn{2}{|c|}{$\begin{array}{c}\text { Température } \\
\left({ }^{\circ} \mathrm{C}\right)\end{array}$} & \multicolumn{2}{|c|}{$\begin{array}{l}\text { Humidité } \\
\text { relative } \\
(\%)\end{array}$} & \multicolumn{2}{|c|}{$\begin{array}{l}\text { Vitesse du } \\
\text { vent }(\mathrm{m} / \mathrm{s})\end{array}$} \\
\hline & Début & Fin & Début & Fin & Début & Fin & Début & Fin & Début & Fin & Début & Fin \\
\hline $15 / 07 / 2016$ & $12 \mathrm{~h} 47$ & $14 \mathrm{~h} 57$ & 1025,9 & 1025,3 & 0 & 0 & 20,8 & 22,1 & 50 & 39 & 15 & 13 \\
\hline $18 / 07 / 2016$ & $13 \mathrm{~h} 41$ & $15 \mathrm{~h} 40$ & 1019,5 & 1018,7 & 0 & 0 & 31,4 & 33,8 & 36 & 28 & 5 & 3 \\
\hline $16 / 08 / 2016$ & $13 \mathrm{~h} 32$ & $15 \mathrm{~h} 14$ & 1013,4 & 1012,6 & 0 & 0 & 30,3 & 32,2 & 37 & 32 & 8 & 6 \\
\hline $17 / 08 / 2016$ & $14 \mathrm{~h} 58$ & $16 \mathrm{~h} 30$ & 1010,2 & 1009,4 & 0 & 0 & 27 & 30,3 & 57 & 41 & 5 & 8 \\
\hline $23 / 08 / 2016$ & $13 \mathrm{~h} 33$ & $15 \mathrm{~h} 27$ & 1023 & 1021,8 & 0 & 0 & 27,3 & 29,1 & 44 & 42 & $\mathrm{nc}$ & $\mathrm{nc}$ \\
\hline $24 / 08 / 2016$ & $15 \mathrm{~h} 51$ & $17 \mathrm{~h} 47$ & 1017,1 & 1016,2 & 0 & 0 & 33,2 & 33,6 & 35 & 33 & 5 & 5 \\
\hline $26 / 08 / 2016$ & $13 \mathrm{~h} 30$ & $15 \mathrm{~h} 14$ & 1016,4 & 1015,6 & 0 & 0 & 30,9 & 33,7 & 40 & 33 & 6 & 5 \\
\hline 29/08/2016 & $14 \mathrm{~h} 41$ & $16 \mathrm{~h} 50$ & 1020,4 & 1019,8 & 0 & 0 & 24 & 24,8 & 58 & 49 & 3 & 6 \\
\hline $30 / 08 / 2016$ & $14 \mathrm{~h} 42$ & $16 \mathrm{~h} 50$ & 1019,2 & 1017,7 & 0 & 0 & 26,5 & 28,7 & 49 & 44 & 11 & 8 \\
\hline $31 / 08 / 2016$ & $14 \mathrm{~h} 39$ & $17 \mathrm{~h} 09$ & 1018,8 & 1017,8 & 0 & 0 & 29,9 & 31,8 & 44 & 38 & $\mathrm{nc}$ & 3 \\
\hline $01 / 08 / 2017$ & $15 \mathrm{~h} 23$ & $18 \mathrm{~h} 37$ & 1012,2 & 1013 & 0 & 0 & 29,4 & 30,2 & 52 & 48 & 10 & 3 \\
\hline $26 / 06 / 2018$ & $12 \mathrm{~h} 15$ & $13 \mathrm{~h} 39$ & 1019 & 1018,6 & 0 & 0 & 25 & 26,6 & 49 & 39 & 5 & 8 \\
\hline $03 / 07 / 2018$ & $12 \mathrm{~h} 29$ & $13 \mathrm{~h} 56$ & 1014,1 & 1013,5 & 0 & 0 & 29,3 & 30,5 & 56 & 50 & 6 & 3 \\
\hline $12 / 07 / 2018$ & $12 \mathrm{~h} 25$ & $13 \mathrm{~h} 10$ & 1015,9 & 1015,7 & 0 & 0 & 25,3 & 26,4 & 43 & 38 & 8 & 8 \\
\hline $19 / 07 / 2018$ & $12 \mathrm{~h} 32$ & $14 \mathrm{~h} 45$ & 1014,2 & 1013,2 & 0 & 0 & 29,8 & 31,3 & 42 & 34 & 5 & 3 \\
\hline $22 / 07 / 2019$ & $12 \mathrm{~h} 25$ & $16 \mathrm{~h} 12$ & 1021,1 & 1019 & 0 & 0 & 31,5 & 35,3 & 38 & 34 & 6 & 5 \\
\hline $31 / 07 / 2019$ & $14 \mathrm{~h} 36$ & $16 \mathrm{~h} 31$ & 1018,1 & 1017,1 & 0 & 0 & 24,2 & 25,9 & 47 & 42 & 6 & 10 \\
\hline $13 / 08 / 2019$ & $15 \mathrm{~h} 12$ & 16h03 & 1018,7 & 1018,5 & 0 & 0 & 22,2 & 22,2 & 51 & 51 & 10 & 10 \\
\hline
\end{tabular}

Tableau 5. Mesures micro-météorologiques mobiles réalisées en été en 2019 utilisées pour l'agglomération de Tokyo. Mobile micro-meteorological measurements carried out in summer in 2019 used for the Tokyo metropolitan area.

\begin{tabular}{|c|c|c|c|c|c|c|c|c|c|c|}
\hline \multirow[b]{2}{*}{ Date } & \multicolumn{2}{|c|}{ Heure locale } & \multicolumn{2}{|c|}{$\begin{array}{c}\text { Pression } \\
\text { barométrique }(\mathrm{hPa})\end{array}$} & \multicolumn{2}{|c|}{$\begin{array}{c}\text { Température } \\
\left({ }^{\circ} \mathrm{C}\right)\end{array}$} & \multicolumn{2}{|c|}{$\begin{array}{l}\text { Humidité } \\
\text { relative (\%) }\end{array}$} & \multicolumn{2}{|c|}{$\begin{array}{l}\text { Vitesse du vent (m } \\
\qquad / \mathrm{s})\end{array}$} \\
\hline & Début & Fin & Début & Fin & Début & Fin & Début & Fin & Début & Fin \\
\hline 21/06/2019 & $16 \mathrm{~h} 35$ & $17 \mathrm{~h} 40$ & 1007,8 & 1008,2 & 28 & 25,5 & 64 & 77 & 22 & 19 \\
\hline $22 / 06 / 2019$ & $14 \mathrm{~h} 01$ & $15 \mathrm{~h} 48$ & 1003,5 & 1002,6 & 22,3 & 22,4 & 99 & 98 & 9 & 4 \\
\hline 23/06/2019 & $14 \mathrm{~h} 27$ & $16 \mathrm{~h} 39$ & 1004,8 & 1004,9 & 23,5 & 22,2 & 82 & 81 & 6 & 9 \\
\hline $25 / 06 / 2019$ & $14 \mathrm{~h} 29$ & $17 \mathrm{~h} 15$ & 1013,9 & 1013,6 & 26,4 & 25,2 & 62 & 67 & 4 & 4,3 \\
\hline 26/06/2019 & $14 \mathrm{~h} 49$ & $17 \mathrm{~h} 45$ & 1010,5 & 1009,7 & 27,7 & 26,2 & 66 & 64 & 6,1 & 6,4 \\
\hline 28/06/2019 & $15 \mathrm{~h} 03$ & $18 \mathrm{~h} 31$ & 996,7 & 998,7 & 29,3 & 27,2 & 74 & 81 & 2,9 & 2,5 \\
\hline $30 / 06 / 2019$ & $13 \mathrm{~h} 06$ & $17 \mathrm{~h} 28$ & 994,7 & 996,2 & 22,6 & 22,3 & 95 & 100 & 1,9 & 1,3 \\
\hline 01/07/2019 & $15 \mathrm{~h} 39$ & $18 \mathrm{~h} 16$ & 1003,6 & 1004,4 & 23,6 & 23,1 & 99 & 100 & 1,7 & 2,2 \\
\hline 02/07/2019 & $14 \mathrm{~h} 28$ & $17 \mathrm{~h} 22$ & 1004,6 & 1004,7 & 26,9 & 25,6 & 85 & 84 & 3,8 & 3,1 \\
\hline 03/07/2019 & $15 \mathrm{~h} 05$ & $18 \mathrm{~h} 48$ & 1007,4 & 1006,8 & 26,8 & 25,4 & 76 & 83 & 2,2 & 2,7 \\
\hline 05/07/2019 & $15 \mathrm{~h} 28$ & $19 \mathrm{~h} 25$ & 1003,9 & 1003,3 & 23,3 & 22,5 & 76 & 78 & 1,6 & 3,5 \\
\hline $11 / 07 / 2019$ & $14 \mathrm{~h} 13$ & $17 \mathrm{~h} 25$ & 1010,9 & 1010,3 & 23,1 & 19,4 & 67 & 98 & 2,9 & 3,1 \\
\hline
\end{tabular}


Les ZCL sont ainsi hiérarchisées par groupes de températures de surface homogènes et cohérents, pour l'année entière et par saisons météorologiques (tableaux 2 et 3). Cependant, afin d'approfondir l'étude et d'analyser la variabilité des ZCL à l'échelle de la journée, la procédure de comparaison multiple est également appliquée pour chacune des dates sélectionnées ; la fréquence d'occurrence (\%) de chacune des journées au sein des différents groupes, déterminés par la procédure de classification multiple, est calculée pour l'année entière ainsi que par saison.

Les températures de l'air sont, quant à elles, confrontées aux ZCL uniquement durant la période de l'été, par manque d'échantillons suffisamment robustes aux autres saisons (tableaux 4 et 5). Une analyse générale et par journée fournissant les fréquences est également menée, tout comme pour les températures de surface.

\section{Résultats : températures de surface et de l'air}

Les résultats du test de Kruskall-Wallis suivis de la procédure de comparaison multiple de SteelDwass-Critchlow-Fligner appliqués sur les températures de surface des après-midis sélectionnés, sur les saisons et sur l'ensemble de l'échantillon indiquent des différences significatives entre les zones climatiques locales.

\subsection{Température de surface des deux agglomérations}

\subsubsection{Température de surface sur la métropole de Tokyo}

A l'échelle annuelle et tous épisodes confondus, les résultats montrent que les ZCL de Tokyo se décomposent, lors des après-midis en 9 catégories distinctes de température de surface (figure 4). La catégorie la plus fraîche (catégorie A) est celle occupée par une couverture végétale, à l'exception des zones composées de plantes rases. En effet, la procédure de comparaison multiple classe dans la catégorie la plus fraîche pour l'ensemble de l'année les surfaces en eau (médiane de $20,9^{\circ} \mathrm{C}$ ), les arbres denses $\left(20,6^{\circ} \mathrm{C}\right)$, les sols nus ou sableux $\left(21^{\circ} \mathrm{C}\right)$, les arbres dispersés $\left(21,4^{\circ} \mathrm{C}\right)$ et les sols rocheux ou pavés $\left(21,7^{\circ} \mathrm{C}\right)$. A cette catégorie la plus fraîche se rajoute une catégorie anthropique : le bâti dense de grande hauteur avec une médiane de $21,5^{\circ} \mathrm{C}$. La présence de cette catégorie urbaine dense peut paraître surprenante au premier abord. Cependant, cette relative fraîcheur à l'échelle annuelle peut s'expliquer par le fait que les bâtiments de grandes hauteurs (à partir de 9 étages notamment : ZCL 1 et 4), surtout ceux de Tokyo, empêchent les radiations solaires d'atteindre la voirie et limitent ainsi le réchauffement des surfaces considérées.

La seconde catégorie (B) comprend uniquement le bâti ouvert de grande hauteur (médiane de $22,6^{\circ} \mathrm{C}$ ). Sa position au septième rang des ZCL les plus fraîches peut également s'expliquer par les mêmes raisons que pour le bâti dense de grande hauteur. Le bâti dispersé constitue la troisième catégorie $\left(23,2^{\circ} \mathrm{C}\right)$. A l'échelle annuelle, ce bâti comporte donc des températures médianes légèrement plus élevées que le bâti de grande hauteur, notamment pour les raisons précédemment citées. La végétation rase n'arrive qu'en neuvième position des ZCL avec des températures médianes de $23,5^{\circ} \mathrm{C}$. Il s'agit donc de $1^{\prime}$ 'occupation du sol végétalisée la plus chaude à l'échelle annuelle. En effet, si ces zones peuvent atteindre des températures très froides durant l'hiver par phénomène de rayonnement par exemple, elles peuvent également atteindre des températures très élevées en été lorsqu'elles ne présentent que très peu de végétation active.

Les catégories E, F, G et $\mathrm{H}$ sont respectivement constituées du bâti ouvert de moyenne hauteur $\left(23,5^{\circ} \mathrm{C}\right)$, du bâti ouvert de faible hauteur $\left(24,1^{\circ} \mathrm{C}\right)$, ainsi que du bâti compact de moyenne $\left(23,8^{\circ} \mathrm{C}\right)$ et faible hauteur $\left(24,2^{\circ} \mathrm{C}\right)$. On peut constater deux règles thermiques dans ce classement : le bâti ouvert est plus frais que celui compact, et le bâti de moyenne hauteur est plus frais que celui de faible hauteur pour les raisons déjà évoquées.

Enfin, la catégorie présentant des températures de surface les plus élevées l'après-midi à l'échelle annuelle (I) rassemble logiquement le bâti large de faible hauteur $\left(24,2^{\circ} \mathrm{C}\right)$ tel que les entrepôts ainsi que les zones d'industrie lourde $\left(24,3^{\circ} \mathrm{C}\right)$. Cette position est expliquée par la forte anthropisation de ces secteurs, la relative moindre hauteur des 
bâtiments qui dépassent rarement un étage par rapport aux zones d'habitat, ainsi que par la chaleur anthropique dégagée par les processus industriels, notamment pétrochimiques dans le cas des zones d'industries lourdes, majoritairement situées en bord de mer pour des raisons de fret maritime.

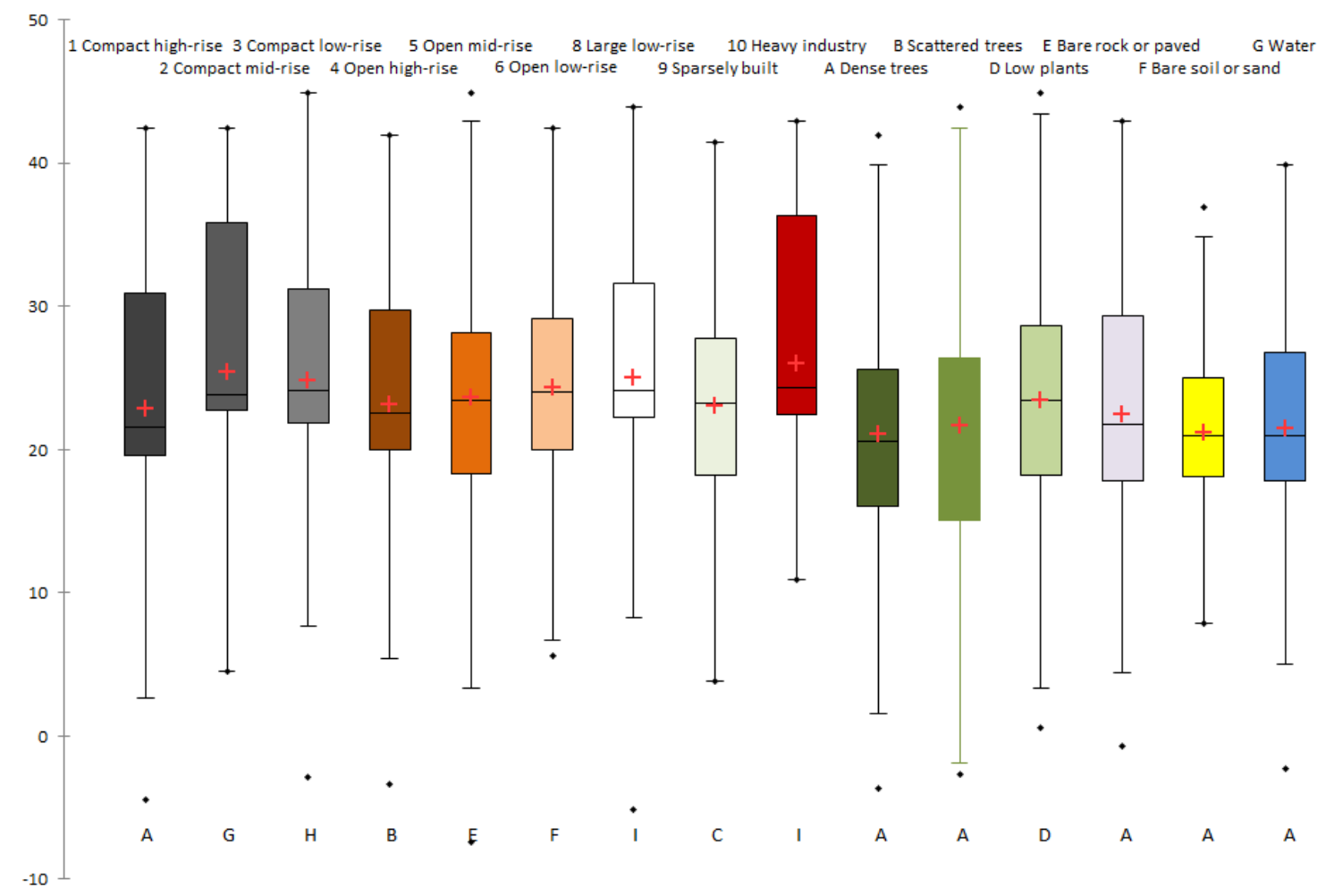

Figure 4. Température de surface (en ${ }^{\circ} \mathrm{C}$ ) pour $15 \mathrm{ZCL}$ de l'agglomération de Tokyo de 2000 à 2019 et classification par groupes suivant la procédure de comparaison multiple de Steel-Dwass-Critchlow-Fligner. Les groupes vont de la classe la plus fraîche $(\mathrm{A})$ à la plus chaude chaude (I). Surface temperature (in ${ }^{\circ} \mathrm{C}$ ) for $15 \mathrm{LCZs}$ in the Tokyo metropolitan area from 2000 to 2019 and classification by groups according to the Steel-Dwass-Critchlow-Fligner Multiple Comparison Procedure. The groups go from the coolest class (A) to the hottest class (I).

Ces résultats sont confirmés lorsqu'on analyse la fréquence des épisodes étudiés en fonction des classes thermiques, d'après la procédure de comparaison multiple, à l'échelle de l'année entière (tableau 6). En effet, en après-midi, les zones en eau sont alors classées à $42 \%$ dans la catégorie la plus fraîche $(\mathrm{A})$ et à $42 \%$ dans les classes $\mathrm{B}$ ou $\mathrm{C}$. Ce même genre de schéma thermique se retrouve également pour les arbres denses, avec $59 \%$ des épisodes classés en A et $39 \%$ en B ou C ; ainsi que pour les arbres dispersés, bien qu'ils ne soient présents qu'à $5 \%$ dans la catégorie A contre $78 \%$ pour les catégories $\mathrm{B}$ et $\mathrm{C}$.

Les zones de sol nu ou de sable, ainsi que les zones de roches nues ou pavées se positionnent également parmi les zones les plus fraîches à l'échelle annuelle avec respectivement 141 et $70 \%$ des journées dans les trois premières catégories les plus fraîches (les totaux supérieurs à $100 \%$ s'expliquent par le fait que la procédure de comparaison multiple de Steel-Dwass-CritchlowFligner peut parfois classer une ZCL dans deux catégories, comme montré sur les figures 4, 5, 6 et 7).

La végétation rase occupe toujours le milieu du classement avec $14 \%$ des journées en catégorie D, $27 \%$ en E, $20 \%$ en F et $5 \%$ en H. Ce comportement thermique est relativement proche du bâti dispersé qui concentre $76 \%$ des journées en catégories D, E et F.

D'une manière générale, à l'échelle de l'année, l'effet de la hauteur du bâti se fait également sentir à travers cette analyse de fréquence par journées. En effet, il peut être constaté que plus la hauteur du bâti est faible, plus il se positionne dans les catégories 
les plus chaudes. Cela est manifeste aussi bien pour le bâti compact que pour le bâti ouvert, les catégories A à D concentrant $90 \%$ des journées pour le bâti compact de grande hauteur, $16 \%$ pour celui de moyenne hauteur et $0 \%$ pour celui de faible hauteur. Parallèlement, les catégories $\mathrm{H}$ à $\mathrm{K}$ regroupent $0 \%$ des journées pour le bâti haut, $43 \%$ pour le moyen et $72 \%$ pour le bâti de faible hauteur.

Le même phénomène est visible pour le bâti ouvert : les catégories A à D concentrent $64 \%$ des journées pour le bâti élevé, $25 \%$ pour celui de moyenne hauteur et $3 \%$ pour celui de faible hauteur. Inversement, les catégories $\mathrm{H}$ à $\mathrm{K}$

Tableau 6. Fréquence (en \%) des 16 ZCL de Tokyo de 2000 à 2019 en fonction des classes déterminées par la procédure de comparaison multiple, de la plus fraîche (A - bleu) à la plus chaude (L - rouge). Frequency (\%) of Tokyo's 16 LCZs from 2000 to 2019 according to the classes determined by the multiple comparison procedure, from coolest ( $A$ - blue) to warmest $(L-r e d)$.

\subsubsection{Température de surface sur la métropole de Lyon}

A l'échelle annuelle, les ZCL de l'agglomération lyonnaise sont composées de sept catégories, selon la procédure de comparaison multiple de SteelDwass-Critchlow-Fligner (figure 5). La catégorie la plus fraîche (A) ne comporte que des zones d'origines naturelles avec, pour débuter, les surfaces en eau qui présentent une médiane de $20,1^{\circ} \mathrm{C}$. Cette catégorie est complétée sans surprise par les arbres denses $\left(22,7^{\circ} \mathrm{C}\right)$ ainsi que par les broussailles $\left(23,6^{\circ} \mathrm{C}\right)$. Cette dernière zone est également classée en zone $\mathrm{B}$, avec les arbres clairsemés $\left(24^{\circ} \mathrm{C}\right)$ et la première occupation du sol anthropisée, le bâti dispersé, qui présente une médiane annuelle de $24,6^{\circ} \mathrm{C}$. Cette catégorie arrive notamment avant la dernière classe de type végétal, regroupent $2 \%$ des journées pour le bâti haut, $7 \%$ pour le moyen et $62 \%$ pour le bâti de faible hauteur.

Il est également intéressant de constater le même phénomène pour les trois catégories de végétation. Plus la végétation est haute, plus la zone sera fraîche. La médiane est de $20,6^{\circ} \mathrm{C}$ pour les arbres denses (catégorie $\mathrm{A}$ ), $23,2^{\circ} \mathrm{C}$ pour les arbres épars (catégorie $\mathrm{A}$ ) et $23,5^{\circ} \mathrm{C}$ pour la végétation rase (catégorie D). A cet effet d'ombrage peut également être rattaché la densité de végétation et la minéralisation inverse relative qui explique ces différences significatives de température.

\begin{tabular}{|lrrrrrrrrrrrr|}
\hline \multicolumn{1}{|c}{ ZCL (\%) } & A & B & C & D & E & F & G & H & I & J & K & L \\
1 Compact high-rise & 25 & 19 & 24 & 22 & 10 & 3 & 2 & 0 & 0 & 0 & 0 & 0 \\
2 Compact mid-rise & 0 & 2 & 2 & 12 & 20 & 10 & 14 & 8 & 20 & 8 & 7 & 0 \\
3 Compact low-rise & 0 & 0 & 0 & 0 & 7 & 14 & 8 & 24 & 32 & 14 & 2 & 0 \\
4 Open high-rise & 2 & 14 & 29 & 19 & 14 & 17 & 5 & 2 & 0 & 0 & 0 & 0 \\
5 Open mid-rise & 0 & 3 & 8 & 14 & 10 & 25 & 32 & 7 & 0 & 0 & 0 & 0 \\
6 Open low-rise & 0 & 0 & 0 & 3 & 14 & 7 & 15 & 31 & 19 & 10 & 2 & 0 \\
8 Large low-rise & 0 & 0 & 0 & 0 & 3 & 10 & 24 & 12 & 22 & 22 & 7 & 0 \\
9 Sparsely built & 0 & 2 & 12 & 27 & 25 & 24 & 5 & 2 & 2 & 2 & 0 & 0 \\
10 Heavy industry & 0 & 2 & 5 & 3 & 7 & 14 & 14 & 17 & 25 & 24 & 24 & 2 \\
A Dense trees & 59 & 34 & 5 & 0 & 0 & 2 & 0 & 0 & 0 & 0 & 0 & 0 \\
B Scattered trees & 5 & 49 & 29 & 8 & 7 & 0 & 0 & 2 & 0 & 0 & 0 & 0 \\
D Low plants & 0 & 2 & 5 & 14 & 27 & 20 & 15 & 5 & 7 & 3 & 2 & 0 \\
E Bare rock or paved & 7 & 31 & 32 & 17 & 10 & 2 & 7 & 2 & 0 & 0 & 0 & 2 \\
F Bare soil or sand & 41 & 63 & 37 & 7 & 5 & 0 & 0 & 0 & 0 & 0 & 2 & 2 \\
G Water & 42 & 20 & 22 & 5 & 8 & 3 & 0 & 0 & 0 & 0 & 0 & 0 \\
\hline
\end{tabular}

la végétation rase, qui est classée dans les catégories $\mathrm{C}$ et $\mathrm{D}$ et qui présente une médiane nettement supérieure de $28^{\circ} \mathrm{C}$. Cette différence de température entre le bâti dispersé et la végétation rase peut s'expliquer par la densité et la hauteur de la végétation en faveur du bâti dispersé. En effet, aux extrémités de l'agglomération lyonnaise, ce genre de bâti est très souvent accompagné de jardins ou de parcs privatifs abondamment pourvus en arbres, avec des routes également souvent bordées d'arbres, contrairement à la végétation rase qui offre une densité végétale moins importante. La catégorie est également constituée par le bâti ouvert de faible hauteur, qui suit directement le bâti dispersé. Il n'est pas surprenant de constater cette stratification thermique directe entre ces deux types de bâti. En effet, ils sont relativement proches et présentent des 
caractéristiques communes telles qu'une faible hauteur des bâtiments ou une végétation environnante relativement développée. Enfin, la dernière zone climatique locale de la classe $\mathrm{C}$ est constituée par le bâti léger et de faible hauteur (i.e. une structure légère non attachée au sol par l'intermédiaire de fondations ou un bâtiment quelconque ouvert sur au moins un côté : $34,1^{\circ} \mathrm{C}$ ), également classée en zone D. Ce bâti regroupe, les structures légères de faible hauteur, souvent temporaires comme les abris de chantier ou les camps de Roms et gens du voyage. La zone D est complétée par le bâti ouvert de moyenne hauteur $\left(33,4^{\circ} \mathrm{C}\right)$. Il convient de remarquer que les deux types de bâti ouvert lyonnais (de faible et de moyenne hauteur) sont classés dans des catégories plus fraîches que les deux types de bâti compact.
La classe $\mathrm{E}$ concentre les zones de roches nues ou pavées $\left(38,8^{\circ} \mathrm{C}\right)$ et de sol nu ou sableux $\left(39,8^{\circ} \mathrm{C}\right)$, avec le bâti compact de faible hauteur dont la médiane atteint $41,3^{\circ} \mathrm{C}$. Celle zone est suivie d'une manière logique par le bâti compact de moyenne hauteur avec une médiane de $41,6^{\circ} \mathrm{C}$ qui finit de constituer la classe F.

Enfin, la classe $\mathrm{G}$ est constituée du bâti large de faible hauteur, tels que les bâtiments industriels de 1'est lyonnais avec une médiane annuelle de $42,8^{\circ} \mathrm{C}$, ainsi que des zones d'industries lourdes comme les installations du couloir de la chimie de Lyon $\left(42,4^{\circ} \mathrm{C}\right)$. La forte minéralisation de ces zones mais surtout la quantité de chaleur anthropique dégagée expliquent cette position dans la classe la plus chaude.

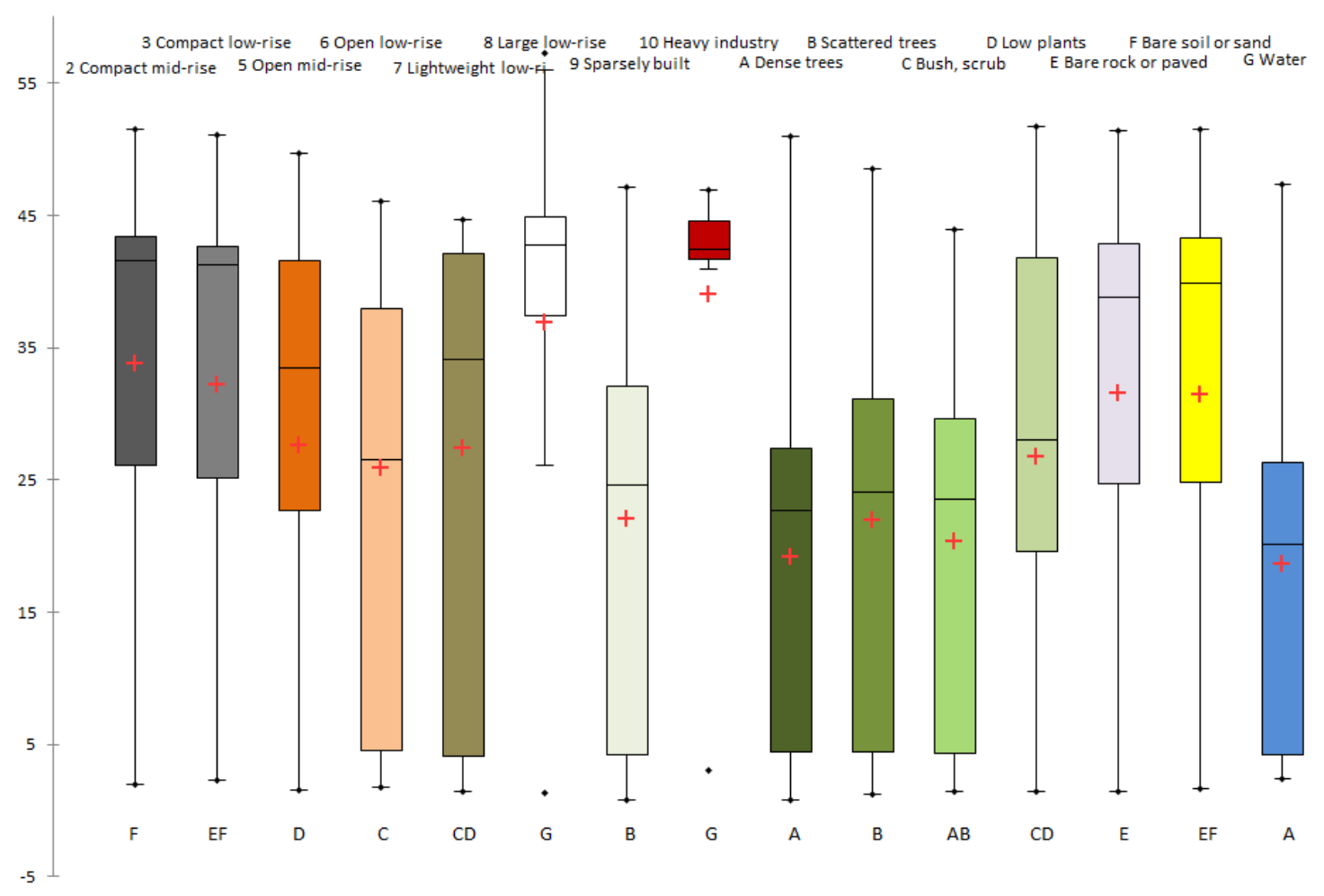

Figure 5. Température de surface des ZCL de l'agglomération de Lyon de 2000 à 2019 et classification par groupes suivant la procédure de comparaison multiple de Steel-Dwass-Critchlow-Fligner. Les groupes vont de la classe la plus fraîche (A) à la plus chaude chaude (G). Surface temperature of the Lyon metropolitan area's ZCL from 2000 to 2019 and classification by groups according to the Steel-Dwass-Critchlow-Fligner multiple comparison procedure. The groups go from the coolest class $(A)$ to the hottest class (G).

L'analyse de la fréquence des journées de passage de Landsat à l'échelle annuelle confirme ces premiers résultats (tableau 7). Les zones en eau sont classées à $98 \%$ dans la catégorie la plus fraîche
(A) et les arbres denses en A (14\%) ou en B (86\%). On remarque un étagement thermique significatif de la végétation, de la plus dense et la plus haute à la plus rase et la plus chaude. En effet, $86 \%$ des zones 
d'arbres denses sont classées en catégorie B, $82 \%$ des arbres espacés sont en catégorie $\mathrm{C}$ tandis que les broussailles glissent légèrement vers la classe suivante avec $30 \%$ en catégorie $\mathrm{D}$, complété par les plantes rases (56\%en catégorie D et $44 \%$ en catégorie E).

Les zones bâties du secteur lyonnais présentent des résultats attendus pour la morphologie urbaine locale. A l'instar de la végétation, on note une stratification thermique marquée. Les zones les moins chaudes sont naturellement le bâti dispersé

Tableau 7. Fréquence (en \%) des ZCL de Lyon en fonction des classes déterminées par la procédure de comparaison multiple, de la plus fraîche (A - bleu) à la plus chaude ( $\mathrm{L}$ - rouge). Frequency (in \%) of Lyon LZs according to the classes determined by the multiple comparison procedure, from the coolest ( $A$ - blue) to the warmest ( $L$ - red). qui est à $72 \%$ dans la classe $\mathrm{D}$, suivi par les structures légères de faible hauteur $(74 \%$ des cas en E). Enfin, le bâti ouvert est moins chaud que celui compact et une hiérarchisation s'opère en fonction de sa densité de végétalisation : le bâti ouvert bas est principalement situé dans les classes $\mathrm{D}$ à $\mathrm{F}$ (102 $\%$ ) alors que le compact l'est principalement dans les classes $\mathrm{F}$ à $\mathrm{G}(111 \%)$. Les zones les plus chaudes lors de l'analyse des journées sont bien le bâti large de faible hauteur et les zones d'industries lourdes avec $44 \%$ chacun dans les classes H et I.

\begin{tabular}{|lccccccccc|}
\hline \multicolumn{1}{|c}{ ZCL (\%) } & A & B & C & D & E & F & G & H & I \\
10 Heavy industry & 0 & 0 & 2 & 2 & 5 & 21 & 26 & 32 & 12 \\
2 Compact mid-rise & 0 & 0 & 0 & 0 & 16 & 21 & 33 & 25 & 5 \\
3 Compact low-rise & 0 & 0 & 2 & 4 & 16 & 44 & 51 & 16 & 4 \\
5 Open mid-rise & 0 & 0 & 0 & 12 & 47 & 42 & 5 & 0 & 0 \\
6 Open low-rise & 0 & 2 & 2 & 21 & 61 & 19 & 0 & 0 & 0 \\
7 Lightweight low-rise & 2 & 2 & 2 & 30 & 74 & 54 & 23 & 11 & 2 \\
8 Large low-rise & 0 & 0 & 0 & 0 & 9 & 23 & 28 & 33 & 11 \\
9 Sparsely built & 0 & 2 & 35 & 72 & 7 & 0 & 0 & 0 & 0 \\
A Dense trees & 14 & 86 & 0 & 0 & 0 & 0 & 0 & 0 & 0 \\
B Scattered trees & 0 & 12 & 82 & 7 & 0 & 0 & 0 & 0 & 0 \\
C Bush, scrub & 0 & 12 & 84 & 30 & 0 & 0 & 0 & 0 & 0 \\
D Low plants & 0 & 2 & 12 & 56 & 44 & 2 & 0 & 0 & 0 \\
E Bare rock or paved & 0 & 2 & 2 & 4 & 28 & 60 & 26 & 7 & 2 \\
F Bare soil or sand & 0 & 0 & 2 & 4 & 30 & 67 & 30 & 5 & 0 \\
G Water & 98 & 2 & 0 & 0 & 0 & 0 & 0 & 0 & 0 \\
\hline
\end{tabular}

l'ensemble les grandes catégories thermiques d'occupation du sol pour les deux agglomérations, eu égard aux différences de morphologies urbaines entre ces deux territoires.

\subsubsection{Température de l'air sur la métropole de Tokyo}

Comme expliqué précédemment dans la partie méthodologique, l'étude des températures de l'air ne concernent que les après-midi des mois estivaux. L'échantillon est composé d'un jeu de 12 mesures itinérantes (tableau 3). Toutes les ZCL ne sont forcément pas représentées en raison des limites inhérentes aux mesures mobiles qui ne permettent pas de parcourir toute l'agglomération, notamment quand il s'agit de zones soumises à des restrictions 
comme les secteurs dédiés à l'industrie lourde où des consignes de sécurité s'appliquent. Ainsi, sur Tokyo, ces secteurs font défaut par rapport à l'analyse des températures de surface, tout comme le sol nu ou sablonneux. Les passages sur les ponts sont considérés comme des zones climatiques locales de surface en eau, notamment le Rainbow bridge. D'une longueur de 570 mètres, ce pont suspendu au-dessus de la baie de Tokyo relie l'agglomération de Tokyo à l'île artificielle d'Odaïba. Il possède en outre des voies piétonnes séparées de la circulation, ce qui limite l'impact de la chaleur anthropique des véhicules qui l'empruntent.

La procédure de classification multiple de SteelDwass-Critchlow-Fligner classe les zones climatiques locales en quatre groupes (figure 6). Il est intéressant de constater que des différences significatives ne se dégagent pas uniquement pour l'analyse par températures de surface mais également à l'aide des températures de l'air. A première vue, cela n'était pas assuré étant donné la plus faible amplitude inter-classe des médianes des températures de $1^{\prime}$ air $\left(5,8^{\circ} \mathrm{C}\right)$ par rapport à celle des températures de surface $\left(7,1^{\circ} \mathrm{C}\right)$.

Le premier groupe est constitué des surfaces en eau, avec une médiane de $25^{\circ} \mathrm{C}$. Un deuxième groupe est constitué des arbres denses et du bâti ouvert de faible hauteur $\left(26,1^{\circ} \mathrm{C}\right.$ et $29,5^{\circ} \mathrm{C}$ respectivement). Ensuite, le troisième groupe est très large puisqu'il regroupe les autres zones climatiques locales, à l'exception de la végétation rase $\left(30,1^{\circ} \mathrm{C}\right)$, du bâti large de faible hauteur $\left(30,2^{\circ} \mathrm{C}\right)$ et du bâti compact de faible hauteur $\left(30,8^{\circ} \mathrm{C}\right)$ qui présentent des températures très élevées et constituent ainsi le dernier groupe.

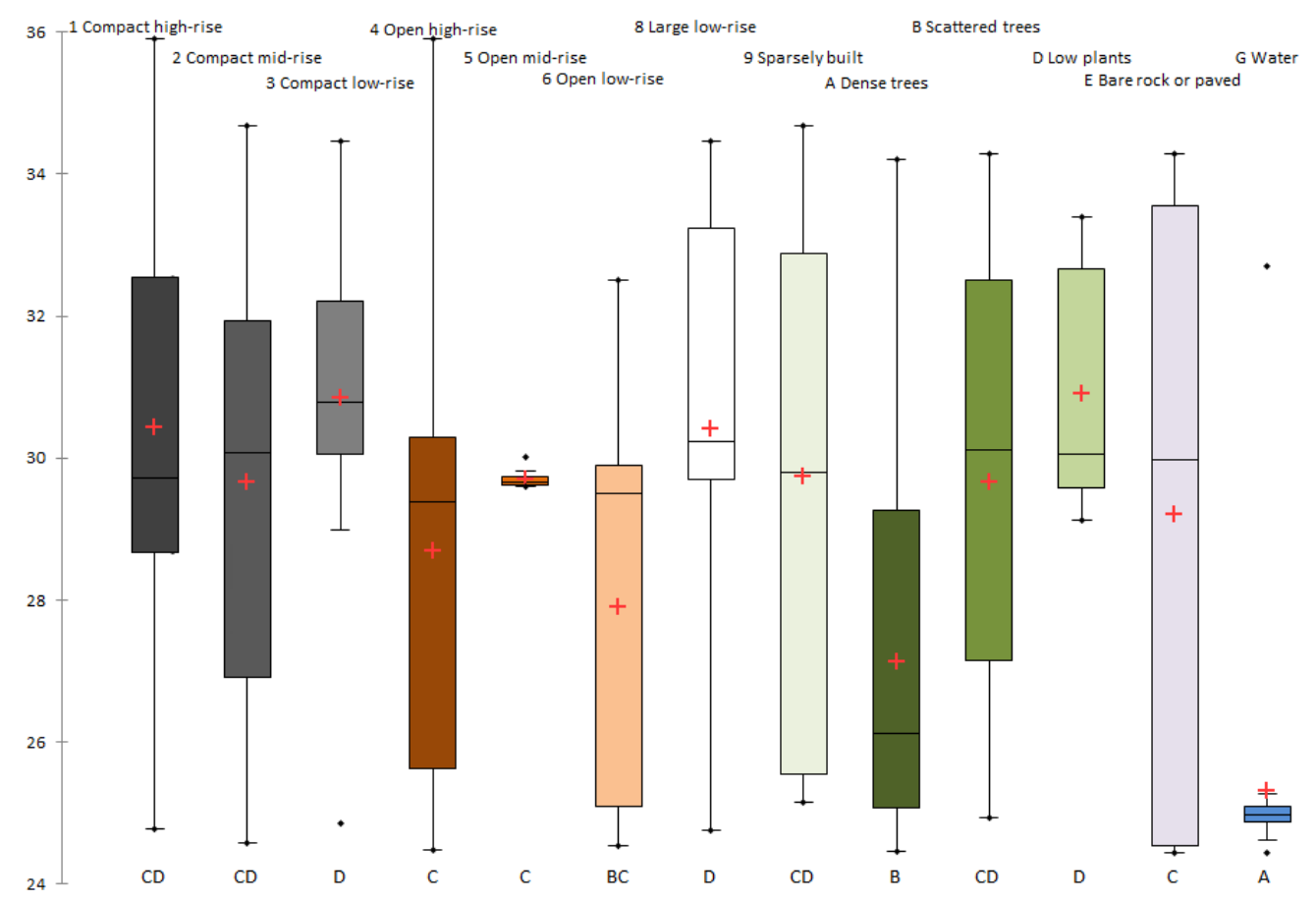

Figure 6. Températures de l'air des ZCL de l'agglomération de Tokyo durant l'été 2019 et classification par groupes suivant la procédure de comparaison multiple. Air Temperatures of the Tokyo Metropolitan LCZs in Summer 2019 and classification by groups according to the multiple comparison procedure.

\subsubsection{Température de l'air sur la métropole de Lyon}

Sur Lyon, sept zones climatiques locales sont analysées lors des 18 campagnes de mesures itinérantes. Tout comme pour Tokyo, la procédure de comparaison multiple permet d'identifier des groupes significativement distincts (figure 7). Les hiérarchisations thermiques précédemment mis en évidence sont globalement respectées, avec les zones climatiques locales végétalisées parmi les 
groupes les plus frais et le bâti plus chaud. Une différence notable est constituée par les zones de roche nue ou minéralisée et les zones de sol nu ou sablonneux.
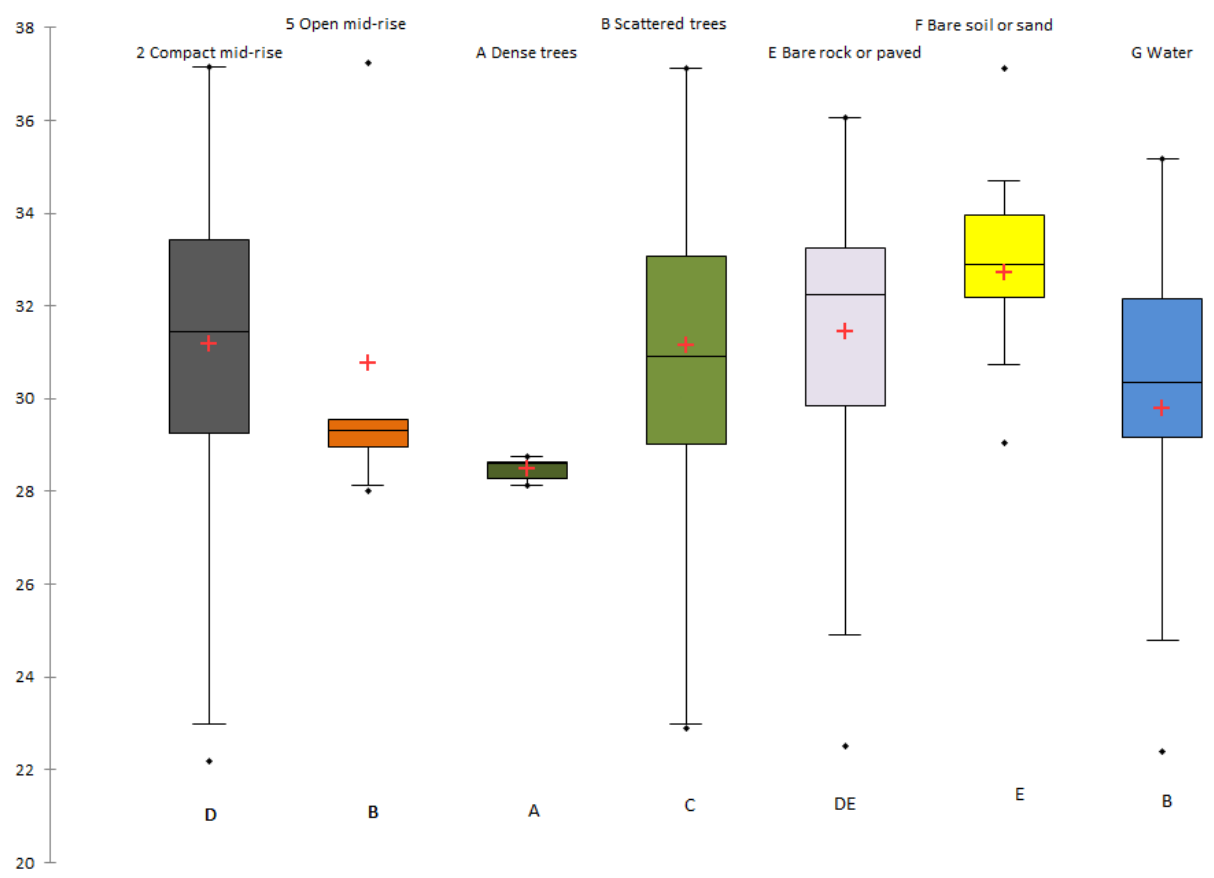

Figure 7. Température de l'air des ZCL de l'agglomération de Lyon en été entre 2016 et 2019 et classification par groupes suivant la procédure de comparaison multiple. Air temperature in the LCZs of the Lyon metropolitan area in Summer from 20016 to 2019 and classification by groups according to the multiple comparison procedure.

En effet, le premier groupe est composé des arbres denses $\left(28,6^{\circ} \mathrm{C}\right.$ de médiane), suivi du groupe B qui comprend les zones en eau $\left(30,3^{\circ} \mathrm{C}\right)$ ainsi que le bâti ouvert de moyenne hauteur $\left(29,3^{\circ} \mathrm{C}\right)$. Il n'est pas vraiment surprenant de ne retrouver les surfaces en eau que dans le groupe B. En effet, à la différence de Tokyo et de son Rainbow bridge, les ponts traversés lors des mesures itinérantes sur Lyon sont de moindre envergure, quelques centaines de mètres (250 mètres environ pour le pont de l'Université ou le pont de la Guillotière). Ils traversent en outre le Rhône et non une baie comme à Tokyo. L'effet rafraîchissant de l'eau s'y fait donc moins ressentir.

La zone des arbres dispersés constituent le troisième groupe avec une médiane de $30,9^{\circ} \mathrm{C}$. Suivent le bâti compact de moyenne hauteur $\left(31,5^{\circ} \mathrm{C}\right)$ et la $\mathrm{ZCL}$ des roches nues et surfaces imperméabilisée $\left(32,2^{\circ} \mathrm{C}\right)$. Enfin, le sol nu ou sableux constitue le groupe dont la température de l'air mesurée est la plus élevée avec une médiane de $32,9^{\circ} \mathrm{C}$. Ces espaces très fortement exposés au soleil et dépourvus de végétation constituent logiquement les zones les plus chaudes de Lyon en été. Les places Bellecour et Terreaux, fortement minéralisées et respectivement pauvres et dénuées de végétation, constituent des lieux bien connus des lyonnais pour leurs températures très élevées en centre-ville. La place Bellecour $\left(46000 \mathrm{~m}^{2}\right)$ est pour moitié recouverte de gore mélangé à de la chaux et d'enrobé pour l'autre moitié. La place des Terreaux $\left(7000 \mathrm{~m}^{2}\right)$ est encore plus minérale car recouverte de dalles de granit sombre et sans végétation.

\section{Discussion}

\subsection{Des caractéristiques de températures de surface similaires sur Lyon et Tokyo avec quelques particularités en fonction du type de bâti}

- Le rôle rafraîchissant de la végétation

L'analyse des températures de surface à l'aide de la procédure de comparaison multiple de SteelDwass-Critchlow-Fligner, aux échelles annuelle et 
saisonnière, complétée par une analyse fréquentielle par journées et par saisons, met en évidence des comportements thermiques contrastés et caractéristiques des diverses zones climatiques. Dans un premier temps, les résultats confirment la plus faible température de surface des surfaces en eau et des zones climatiques locales pourvues en végétation.

- La densité de végétation est déterminante

Les résultats mettent également en évidence le pouvoir rafraîchissant de la densité de la biomasse ainsi que de sa hauteur. Ainsi, les zones d'arbres denses sont globalement les plus fraîches, devant les arbres espacés, les buissons et taillis. Le bâti dispersé fait jeu égal d'un point de vue de la température de surface avec la végétation rase. En effet, ce dernier type présente souvent une forte densité de végétation, au contraire des ZCL de végétation rase qui peuvent offrir peu de biomasse dans le cas de cultures rases, retenant très faiblement l'humidité et ne permettant pas un rafraîchissement par transfert de chaleur latente ou par ombrage.

- Les zones d'activité présentent les températures de surface les plus élevées

Les zones climatiques anthropisées enregistrent naturellement des températures de surface plus élevées. Cependant, des différences significatives et systématiques sont mises en évidence au sein de ces zones. Dans un premier temps, en après-midi, le bâti compact et le bâti ouvert développent des températures de surface inférieures au bâti large de faible hauteur et aux zones d'industries lourdes. Comme expliqué précédemment, cela est dû à la quantité de chaleur anthropique liée à ces zones d'activité, associé à une végétalisation relativement faible ou nulle ( Wicki et al., 2017 ; Dong et al., 2017 ; Shandas et al., 2019). Nous remarquons également que la variabilité intra-classe de ces deux zones climatiques est très faible pour la métropole lyonnaise, notamment pour les zones industrielles lourdes. Ces zones présentent des températures de surface toujours relativement très élevées qui ne varient pas beaucoup avec la saison, en raison de la chaleur anthropique dégagée par les processus industriels. Pour le territoire de Tokyo, cette variabilité reste du même ordre que celle des autres zones climatiques.

- Le bâti ouvert logiquement plus frais que le bâti compact

A Lyon, le bâti ouvert reste plus frais que le bâti compact, aussi bien à l'échelle annuelle que l'été. Cela est encore une fois expliquée par la fraction de végétation disponible (Rizwan et al., 2008 ; Makido et al., 2019 ; Masoudi et Tan, 2019). Cette tendance est moins marquée pour l'agglomération de Tokyo, même si cela reste également valable.

- Des différences dans l'effet de la hauteur des bâtiments

Une différence notable peut être remarquée lorsque l'on s'intéresse à la hauteur du bâti compact et ouvert des deux agglomérations. Dans la métropole lyonnaise, plus le bâti est haut, plus celuici sera d'une température de surface élevée. Cela est nettement visible à l'échelle annuelle et en été notamment. Le phénomène inverse se manifeste à Tokyo à toutes les échelles temporelles d'étude, en hiver comme en été. Les différences constatées entre les agglomérations de Lyon et de Tokyo proviendraient des morphologies urbaines propres aux deux mégapoles. En effet, à Tokyo, la hauteur et la densité du bâti étant nettement plus élevées que sur Lyon, les bâtiments ont donc une plus grande propension à intercepter les rayons solaires et ainsi, à créer un rafraîchissement en projetant leur ombre (Zhang et al., 2017 ; Sun et al., 2019 ; Yu et al., 2019). Cela a déjà été notifié dans différentes études, surtout dans les pays du sud-est asiatique (Lin et al., 2010 ; Chan, 2012 ; Martinelli et al., 2015). Ce phénomène n'est pas un objet d'étude récent, les premières publications remontant aux années 1990 et 2000 (Oke, 1992 ; Lam, 2000). Il est cependant toujours très difficile d'évaluer quantitativement l'impact sur le bilan énergétique à l'échelle micro-locale (Lin et al., 2017 ; Yu et al., 2019). 
- Des différences entre agglomérations pour les températures de surface des ZCL nues ou minéralisées

Il existe des différences significatives dans les températures de surface des ZCL E (roche nue ou minéralisée) et $\mathrm{F}$ (sol nu ou sablonneux). Leurs températures sont moins élevées sur l'agglomération de Tokyo, en toutes saisons, par rapport aux températures des autres ZCL. Les températures de ces deux classes se situent ici plutôt au niveau de celles végétalisées. Sur Lyon, les températures de surface des classes $\mathrm{E}$ et $\mathrm{F}$ se rangent plutôt aux côtés des valeurs des ZCL composées du bâti compact ou ouvert. Plusieurs pistes peuvent être avancées pour expliquer cette différence de comportement thermique.

Dans un premier temps, à Tokyo, la classe des sols nus ou sablonneux se localisent préférentiellement dans les lits majeurs des cours d'eau. Ces terrains se trouvent ainsi à proximité d'une source d'humidité et bénéficient également de l'ombrage de la ripisylve. Cela pourrait expliquer ces températures relativement basses par rapport à celles de Lyon car, sur ce territoire, il s'agit plus de gravières et de carrières produisant des granulats (ainsi que la moitié de la surface de la place Bellecour).

Dans un second temps, la classe de roche nue ou des surfaces minéralisées bénéficieraient à Tokyo d'un ombrage préférentiel, en raison de la densité des bâtiments et de leur hauteur (avec un nombre d'étage fréquemment supérieur à $9:$ ZCL 1 et 4), ce qui n'est pas le cas sur l'agglomération lyonnaise. En outre, sur Lyon, ces zones sont principalement constituées par les larges espaces de transit ferroviaire du $2^{\text {ème }}$ et du sud du $7^{\text {ème }}$ arrondissement, avec environ $500000 \mathrm{~m}^{2}$ environ pour ce dernier, et du couloir de la chimie, dont les constituants (rails et ballast) peuvent atteindre des températures de surface très élevées. Il y a aussi le cas de la place des Terreaux, dépourvue de toute végétation et qui demeure un des 'hot spots' de l'agglomération

\footnotetext{
1 www.leprogres.fr/environnement/2020/08/09/en-images-avec-la-caniculeles-lyonnais-cherchent-la-fraîcheur
}

lyonnaise en période estivale, même si des petites fontaines $\mathrm{y}$ ont récemment été installées au printemps dernier ${ }^{12}$.

\subsection{Les températures de l'air des ZCL précisent les résultats issus des températures de surface}

- Des températures de l'air élevées sur Tokyo pour la végétation rase

Les températures de l'air sont en adéquation avec les résultats obtenus lors de l'analyse des températures de surface pour les catégories les plus fraîches, i.e. les zones en eau et les arbres denses, ainsi que les catégories les plus chaudes, i.e. le bâti compact de faible hauteur et le bâti large de faible hauteur. Cependant, il est intéressant de remarquer une différence notable : la végétation rase se hisse parmi les ZCL du groupe le plus chaud, en raison d'une température de l'air relativement élevée. Cette zone climatique était plutôt située dans les groupes les plus frais pour la température de surface, même en été. Cette position s'explique par la faible fraction de végétation présente dans cette zone ainsi que par une exposition solaire maximale, à la différence de celles constituées d'arbres denses ou d'arbres dispersés positionnées logiquement dans les groupes les plus frais. Ainsi, la stratification thermique en fonction de la biomasse est également vérifiée pour les températures de l'air (Rizwan et al., 2008 ; Voelkel et Shandas, 2017 ; Makido et al., 2019).

- Une confirmation de la hiérarchisation thermique du bâti en fonction de la densité de végétation

Il a été montré à l'aide de l'analyse portant sur les températures de surface que la densité de végétation à l'intérieur des zones climatiques de bâti compact et ouvert oriente leur comportement thermique. Cela est également le cas pour les températures de l'air. En effet, le bâti ouvert, qu'il soit de moyenne ou de faible hauteur, possède des températures de l'air inférieures au bâti compact,

\footnotetext{
${ }^{2}$ met.grandlyon.com/travaux-terreaux-octobre-2018/
} 
sur Tokyo comme sur Lyon.

- La stratification thermique de Tokyo liée à la hauteur des bâtiments compacts est confirmée par les températures de l'air

L'analyse des températures de surface de Tokyo a également révélé un gradient thermique décroissant avec la hauteur des bâtiments, les bâtiments de plus de 10 étages (ZCL 1 et 4) ayant des températures de surface plus fraîches que les bâtiments de 4 à 9 étages (ZCL 2 et 5), eux-mêmes plus frais que les bâtiments de trois étages maximum (ZCL 3 et 6). Cette observation est également vérifiée pour les bâtiments compacts de Tokyo (le bâti traversé à Lyon ne présentant que des hauteurs moyennes de 4 à 9 étages). En effet, le bâti de faible hauteur (3 étages maximum) présente la médiane la plus élevée avec une température de $30,8^{\circ} \mathrm{C}$ et se classe dans le groupe le plus chaud (avec le bâti large de faible hauteur également), tandis que celui de moyenne hauteur (4 à 9 étages) a une médiane de $30,1^{\circ} \mathrm{C}$ et celui de forte hauteur (à partir de 10 étages) une médiane de $29,7^{\circ} \mathrm{C}$. Cependant, cela n'est pas vérifié pour le bâti ouvert, qui présente des températures médianes relativement proches, quelle que soit sa hauteur, dans une amplitude de $0,3^{\circ} \mathrm{C}\left(29,4^{\circ} \mathrm{C} ; 29,7^{\circ} \mathrm{C}\right.$ et $29,5^{\circ} \mathrm{C}$ respectivement pour le bâti de grande, moyenne et faible hauteurs). Cela est probablement lié au rôle de la végétation qui vient tempérer l'effet d'ombrage des bâtiments.

\section{Conclusion}

Les zones climatiques locales (ZCL) sont un outil de plus en plus utilisé dans les problématiques d'aménagement grâce à leur universalité et à leur facilité d'obtention, que ce soit par photointerprétation pour les petits territoires comme l'agglomération lyonnaise ou par classification assistée pour les plus grands comme celle de Tokyo. Il est cependant nécessaire de les valider localement à l'aide de mesures de température de surface et de l'air, avec des analyses à l'échelle annuelle et saisonnière.

Les températures de l'air ont cependant une plage beaucoup plus faible que celles de surface. En effet, pour une même journée, il est très rare de trouver des différences de température de l'air de $3^{\circ} \mathrm{C}$ alors que celles de surface peuvent dépasser $30^{\circ} \mathrm{C}$. Cependant, la procédure de classification multiple a tout de même mis en évidence des groupes significativement différents en fonction de la température de l'air.

D'une manière assez logique, parmi les zones les plus chaudes, nous retrouvons les zones d'activité, les zones industrielles et celles de bâti alors que les zones les plus fraîches sont marquées par une densité de végétation significative. Des nuances ont également été mises en évidence. Dans un premier temps, la densité de végétation et la quantité de biomasse produisent un gradient thermique décroissant. En outre, la hauteur du bâti influe sur la température. L'ombre projetée des bâtiments aura un effet rafraichissant sur sa zone climatique locale. Enfin, la chaleur anthropique des zones d'activités ou des industries lourdes se manifestent aussi bien lors de l'analyse des températures de surfaces que des températures de l'air.

En termes de limites, il peut être remarqué que la classification des ZCL a été effectuée sur Lyon par photo-interprétation alors qu'une classification assistée random forest a été utilisée pour Tokyo. Bien que cette classification ait fourni de très bons résultats lors de la confrontation avec le terrain, elle ne peut égaler une photo-interprétation (qui présente elle aussi des limites certaines). En outre, cette étude repose sur une exploitation de données satellitaires de 2000 à 2019 confrontée aux ZCL obtenues en 2018 pour Lyon et en 2017 pour Tokyo. Des modifications d'usage des sols ont eu lieu durant ces années ce qui peut limiter la représentativité des résultats. Une solution serait de réaliser une classification de ZCL par année mais cela s'avérerait très chronophage. L'heure de passage de Landsat au-dessus des deux territoires d'étude présente aussi quelques limites. En effet, le satellite passe au-dessus de l'agglomération lyonnaise à 10h20 UTM (donc $11 \mathrm{~h} 30$ ou $12 \mathrm{~h} 30$ localement) mais à 01h15 UTM à Tokyo (donc 10h15 localement). De plus, bien que cette méthode soit transposable à d'autres villes, les résultats 
concernant les températures de l'air ne le sont que pour les après-midi. Enfin, les mesures mobiles ont été effectuées en vélo à vitesse réduite sur Lyon et à pied sur Tokyo pour des raisons pratiques, mais nous estimons que cela n'a pas eu d'impact quantifiable sur les mesures.

En termes de perspectives, les campagnes de mesures itinérantes sont à poursuivre afin d'étoffer l'échantillon de ZCL traversées. Cependant, il nous semble difficile de parvenir à faire des mesures dans les zones d'industries lourdes, à Lyon comme à Tokyo, ces dernières ayant des fréquentations contrôlées par mesure de sécurité. Il serait également intéressant de traiter les mesures mobiles effectuées en matinée et en soirée car les gradients thermiques au sein d'une agglomération ne sont pas stationnaires. Cela pourrait également être complété par une analyse des températures de surface nocturne, Tokyo bénéficiant en effet d'un passage de Landsat aux alentours de $21 \mathrm{~h} 30$.

Remerciements : Nous tenons à remercier nos collègues du Tokyo Institute of Technology pour les collaborations sur les mesures mobiles et les zones climatiques locales, l'ambassade de France au Japon ainsi que l'Ecole Urbaine de Lyon pour leurs soutiens logistiques.

\section{Références}

Alexander P. J., Mills G., Fealy R., 2015. Using LCZ data to run an urban energy balance model. Urban Climate, 13, 1437. https://doi.org/10.1016/j.uclim.2015.05.001

Alonso L., Renard F., 2020. A comparative study of the physiological and socio-economic vulnerabilities to heat waves of the population of the Metropolis of Lyon (France) in a climate change context. International Journal of Environmental Research and Public Health, 17, 1004. https://doi.org/10.3390/ijerph17031004

Alonso L., Renard F., 2019. Integrating satellite-derived data as spatial predictors in multiple regression models to enhance the knowledge of air temperature patterns. Urban Science, 3, 101. https://doi.org/10.3390/urbansci3040101

Azevedo J. A., Chapman L., Muller C. L., 2016. Quantifying the daytime and night-time Urban Heat Island in Birmingham, UK: A comparison of satellite derived land surface temperature and high resolution air temperature observations. Remote Sensing, 8, 153. https://doi.org/10.3390/rs8020153

Barreca A., Clay K., Deschenes O., Greenstone M., Shapiro J.
S., 2016. Adapting to climate change: The remarkable decline in the US temperature-mortality relationship over the Twentieth Century. Journal of Political Economy, 124, $105-$ 159. https://doi.org/10.1086/684582

Bechtel B., Alexander P. J., Böhner J., Ching J., Conrad O., Feddema J., Mills G., See L., Stewart I., 2015. Mapping Local Climate Zones for a worldwide database of the form and function of cities. ISPRS International Journal of GeoInformation, 4, 199-219. https://doi.org/10.3390/ijgi4010199

Bechtel B., Demuzere M., Sismanidis P., Fenner D., Brousse O., Beck C., Van Coillie F., Conrad O., Keramitsoglou I., Middel A., Mills G., Niyogi D., Otto M., See L., Verdonck M.-L., 2017. Quality of crowdsourced data on urban morphology — The Human Influence Experiment (HUMINEX). Urban Science, 1, https://doi.org/10.3390/urbansci1020015

Bobb J. F., Peng R. D., Bell M. L., Dominici F., 2014. Heatrelated mortality and adaptation to heat in the United States. Environ. Health Perspect., 122, 811-816. https://doi.org/10.1289/ehp.1307392

Box G. E. P., 1953. Non-normality and tests on variances. Biometrika, 40, 318-335. https://doi.org/10.1093/biomet/40.34.318

Breiman L., 2001. Random Forests. Machine Learning, 45, 532. https://doi.org/10.1023/A:1010933404324

Breiman L., Friedman J. H., Olshen R. A., Stone C. J., 2017. Classification and regression trees. Chapman and Hall/CRC ed., New-York.

Brousse O., Martilli A., Foley M., Mills G., Bechtel B., 2016. WUDAPT, an efficient land use producing data tool for mesoscale models? Integration of urban LCZ in WRF over Madrid. Urban Climate, 17, 116-134. https://doi.org/10.1016/j.uclim.2016.04.001

Burke M., González F., Baylis P., Heft-Neal S., Baysan C., Basu S., Hsiang S., 2018. Higher temperatures increase suicide rates in the United States and Mexico. Nature Climate Change, 8, 723-729. https://doi.org/10.1038/s41558-0180222-X

Burke M., Gonzalez F., Baylis P., Heft-Neal S., Baysan C., Hsiang S., 2020. Reply to: Temporal displacement, adaptation and the effect of climate on suicide rates. Nature Climate Change, 1-3. https://doi.org/10.1038/s41558-020-0792-2

Chan A. L. S., 2012. Effect of adjacent shading on the thermal performance of residential buildings in a subtropical region. Applied Energy, 92, 516-522. https://doi.org/10.1016/j.apenergy.2011.11.063

Conover W. J., 1998. Practical nonparametric statistics. 3rd Edition, Wiley, 592 pages.

IPCC, 2014. Climate Change 2014: Synthesis Report. Contribution of Working Groups I, II and III to the Fifth Assessment Report of the Intergovernmental Panel on Climate Change, Core Writing Team, R.K. Pachauri and L.A. Meyer (eds.), IPCC. ed. Geneva, Switzerland. 
Della Marta P. M., Haylock M. R., Luterbacher J., Wanner H., 2007. Doubled length of western European summer heat waves since 1880. Journal of Geophysical Research: Atmospheres, 112. https://doi.org/10.1029/2007JD008510

Demuzere, M., Bechtel, B., Middel, A., Mills, G., 2019. Mapping Europe into local climate zones. PLOS ONE, 14, e0214474. https://doi.org/10.1371/journal.pone.0214474

Desai M. S., Dhorde A. G., 2018. Trends in thermal discomfort indices over western coastal cities of India. Theor. Appl. Climatol., 131, 1305-1321. https://doi.org/10.1007/s00704017-2042-8

Dousset B., Gourmelon F., Laaidi K., Zeghnoun A., Giraudet E., Bretin P., Mauri E., Vandentorren S., 2011. Satellite monitoring of summer heat waves in the Paris metropolitan area. International Journal of Climatology, 31, 313-323. https://doi.org/10.1002/joc.2222

Dunn O. J., 1964. Multiple comparisons using rank sums. Technometrics, 6, 241-252.

Fink A. H., Brücher T., Krüger A., Leckebusch G. C., Pinto J. G., Ulbrich U., 2006. The 2003 European summer heatwaves and drought-synoptic diagnosis and impacts. Weather, 59, 209-216. https://doi.org/10.1256/wea.73.04

Fligner M. A., 1984. A note on two-sided distribution-free treatment versus control multiple comparisons. Journal of the American Statistical Association, 79, 208-211.

Foissard X., Dubreuil V., Quénol H., 2019. Defining scales of the land use effect to map the urban heat island in a mid-size European city: Rennes (France). Urban Climate, 29, 100490. https://doi.org/10.1016/j.uclim.2019.100490

Gammans M., 2020. Temporal displacement, adaptation and the effect of climate on suicide rates. Nature Climate Change, 1-3. https://doi.org/10.1038/s41558-020-0791-3

Grömping U., 2009. Variable importance assessment in regression: Linear regression versus Random Forest. The American Statistician, 63, 308-319. https://doi.org/10.1198/tast.2009.08199

Hajat S., Kosatky T., 2010. Heat-related mortality: a review and exploration of heterogeneity. J. Epidemiol. Community Health, 64, 753-760. https://doi.org/10.1136/jech.2009.087999

Hollander M., Wolfe D. A., 1999. Nonparametric statistical methods. Second edition, John Wiley and Sons ed., NewYork, 816 pages.

IPCC, 2013.. Contribution Climate Change 2013: The Physical Science Basis of Working Group I to the Fifth Assessment Report of the Intergovernmental Panel on Climate Change. Stocker T.F., D. Qin, G.-K. Plattner, M. Tignor, S. K. Allen, J. Boschung, A. Nauels, Y. Xia, V. Bex and P. M. Midgley, ed. Cambridge University Press, Cambridge.

Jenerette G. D., Harlan S. L., Buyantuev A., Stefanov W. L., Declet-Barreto J., Ruddell B. L., Myint S. W., Kaplan S., Li X., 2016. Micro-scale urban surface temperatures are related to land-cover features and residential heat related health impacts in Phoenix, AZ USA. Landscape Ecol., 31, 745-760. https://doi.org/10.1007/s10980-015-0284-3

Jones S., Reinke K., 2009. Innovations in remote sensing and photogrammetry. Leading edge, applied research in the area of Remote Sensing and Photogrammetry. Springer Science \& Business Media, Jones et Reinke (Eds.), Publisher SpringerVerlag Berlin Heidelberg, 468 p., DOI 10.1007/978-3-540-

Kakamu T., Wada K., Smith D. R., Endo S., Fukushima T., 2017. Preventing heat illness in the anticipated hot climate of the Tokyo 2020 Summer Olympic Games. Environ. Health Prev. Med., 22, 68. https://doi.org/10.1186/s12199-017-0675$\mathrm{y}$

Kosaka E., Iida A., Vanos J., Middel A., Yokohari M., Brown R., 2018. Microclimate variation and estimated heat stress of runners in the 2020 Tokyo Olympic marathon. Atmosphere, 9 , 192. https://doi.org/10.3390/atmos9050192

Kovats R. S., Hajat S., 2008. Heat stress and public health: a critical review. Annu. Rev. Public Health, 29, 41-55. https://doi.org/10.1146/annurev.publhealth.29.020907.09084 3

Laaidi K., Zeghnoun A., Dousset B., Bretin P., Vandentorren S., Giraudet E., Beaudeau P., 2012. The impact of heat islands on mortality in Paris during the August 2003 heat wave. Environ. Health Perspect., 120, 254-259. https://doi.org/10.1289/ehp.1103532

Lam J. C., 2000. Shading effects due to nearby buildings and energy implications. Energy Conversion and Management, 41, 647-659. https://doi.org/10.1016/S0196-8904(99)00138-7

Landis J. R., Koch G. G., 1977. A one-way components of variance model for categorical data. Biometrics, 33, 671-679. https://doi.org/10.2307/2529465

Leconte F., Bouyer J., Claverie R., Pétrissans M., 2017. Analysis of nocturnal air temperature in districts using mobile measurements and a cooling indicator. Theor. Appl. Climatol., 130, 365-376. https://doi.org/10.1007/s00704-016-1886-7

Lin P., Lau S. S. Y., Qin H., Gou Z., 2017. Effects of urban planning indicators on urban heat island: a case study of pocket parks in high-rise high-density environment. Landscape and Urban Planning, 168, 48-60. https://doi.org/10.1016/j.landurbplan.2017.09.024

Lin T.-P., Matzarakis A., Hwang R.-L., 2010. Shading effect on long-term outdoor thermal comfort. Building and Environment. International Symposium on the Interaction between Human and Building Environment, Special Issue, Section 45, 213-221. https://doi.org/10.1016/j.buildenv.2009.06.002

Martinelli L., Lin T.-P., Matzarakis A., 2015. Assessment of the influence of daily shadings pattern on human thermal comfort and attendance in Rome during summer period. Building and Environment, 92, 30-38. https://doi.org/10.1016/j.buildenv.2015.04.013

Meehl G. A., Tebaldi C., 2004. More intense, more frequent, and longer lasting heat waves in the 21st Century. Science, 305, 994-997. https://doi.org/10.1126/science.1098704

Metzger K., Ito K., Matte T., 2010. Summer heat and mortality 
in New York City: How hot is too hot? Environmental Health Perspectives, 118, 80-86. https://doi.org/10.1289/ehp.0900906

Molnár G., Gyöngyösi A. Z., Gál T., 2019. Integration of an LCZ-based classification into WRF to assess the intra-urban temperature pattern under a heatwave period in Szeged, Hungary. Theor. Appl. Climatol., 138, 1139-1158. https://doi.org/10.1007/s00704-019-02881-1

Nayak S. G., Shrestha S., Kinney P. L., Ross Z., Sheridan S. C., Pantea C. I., Hsu W. H., Muscatiello N., Hwang S. A., 2017. Development of a heat vulnerability index for New York State. Public Health, 161, https://doi.org/10.1016/j.puhe.2017.09.006

Ok V., 1992. A procedure for calculating cooling load due to solar radiation: the shading effects from adjacent or nearby buildings. Energy and Buildings, 19, 11-20. https://doi.org/10.1016/0378-7788(92)90032-C

Pascal M., Wagner V., Corso M., Laaidi K., Le Tertre A., 2019. Évolution de l'exposition aux canicules et de la mortalité associée en France métropolitaine entre 1970 et 2013. Santé Publique France, Saint-Maurice : Santé publique France, 2019, 69 p., ISBN-NET : 979-10-289-0488-3

Patz J. A., Campbell-Lendrum D., Holloway T., Foley J. A., 2005. Impact of regional climate change on human health. Nature, 438, 310-317. https://doi.org/10.1038/nature04188

Petitti D., Hondula D., Shuo Y., Harlan S., Chowell G., 2016. Multiple trigger points for quantifying heat-health impacts: New evidence from a hot climate. Environmental Health Perspectives, 124, 176-183. https://doi.org/10.1289/ehp.1409119

Qaid A., Lamit H. B., Ossen D. R., Rasidi M. H., 2018. Effect of the position of the visible sky in determining the sky view factor on micrometeorological and human thermal comfort conditions in urban street canyons. Theor. Appl. Climatol., 131, 1083-1100. https://doi.org/10.1007/s00704-016-2023-3

Renard F., Alonso L., Fitts Y., Hadjiosif A., Comby J., 2019. Evaluation of the effect of urban redevelopment on surface Urban Heat Islands. Remote Sensing, 11, 299. https://doi.org/10.3390/rs11030299

Richard Y., Emery J., Dudek J., Pergaud J., Chateau-Smith C., Zito S., Rega M., Vairet T., Castel T., Thévenin T., Pohl B., 2018. How relevant are local climate zones and urban climate zones for urban climate research? Dijon (France) as a case study. Urban Climate, 26, 258-274. https://doi.org/10.1016/j.uclim.2018.10.002

Robine J.-M., Cheung S. L. K., Le Roy S., Van Oyen H., Griffiths C., Michel J.-P., Herrmann F. R., 2008. Death toll exceeded 70,000 in Europe during the summer of 2003. Comptes Rendus Biologies, Dossier: Nouveautés en cancérogenèse / New developments in carcinogenesis 331, 171-178. https://doi.org/10.1016/j.crvi.2007.12.001

Seltenrich N., 2015. Between extremes: Health effects of heat and cold. Environmental Health Perspectives, 123, A275A279. https://doi.org/10.1289/ehp.123-A275

Shapiro S. S., Wilk B., 1965. An analysis of variance test for normality (complete samples). Biometrika, 52 (3-4), 591-611.
Smargiassi A., Goldberg M. S., Plante C., Fournier M., Baudouin Y., Kosatsky T., 2009. Variation of daily warm season mortality as a function of micro-urban heat islands. J. Epidemiol. Community Health, 63, 659-664. https://doi.org/10.1136/jech.2008.078147

Sobrino J. A., Jiménez-Muñoz J. C., Paolini L., 2004. Land surface temperature retrieval from LANDSAT TM 5. Remote Sensing of Environment, 90, 434-440. https://doi.org/10.1016/j.rse.2004.02.003

Sobrino, J.A., Oltra-Carrió, R., Sòria, G., Jiménez-Muñoz, J.C., Franch, B., Hidalgo, V., Mattar, C., Julien, Y., Cuenca, J., Romaguera, M., Gómez, J.A., Miguel, E.D., Bianchi, R., Paganini, M., 2013. Evaluation of the surface urban heat island effect in the city of Madrid by thermal remote sensing. International Journal of Remote Sensing, 34, 3177-3192. https://doi.org/10.1080/01431161.2012.716548

Stewart I. D., Oke T. R., 2012. Local Climate Zones for urban temperature studies. Bull. Amer. Meteor. Soc., 93, 1879-1900. https://doi.org/10.1175/BAMS-D-11-00019.1

Suzuki-Parker A., Kusaka H., Yamagata Y., 2015. Assessment of the impact of metropolitan-scale urban planning scenarios on the moist thermal environment under global warming: A study of the Tokyo metropolitan area using regional climate modeling. Advances in Meteorology, e693754. https://doi.org/10.1155/2015/693754

Tan J., Zheng Y., Tang X., Guo C., Li L., Song G., Zhen X., Yuan D., Kalkstein A. J., Li F., Chen H., 2010. The urban heat island and its impact on heat waves and human health in Shanghai. Int. J. Biometeorol., 54, 75-84. https://doi.org/10.1007/s00484-0090256-X

United Nations, Department of Economic and Social Affairs, Population Division, 2019. World Population Prospects 2019: Highlights. No. ST/ESA/SER.A/423.

United Nations, Department of Economic and Social Affairs, Population Division, 2018. The World's Cities in 2018-Data Booklet. No. ST/ESA/ SER.A/417, United Nations.

United Nations, Department of Economic and Social Affairs, Population Division, 2014. World Urbanization Prospects: The 2014 Revision, Highlights. ST/ESA/SER.A/352.

United Nations Population Fund, 2007. State of World Population. Éditeur: UNFPA, New-York, USA, 108 p., ISBN: 978-0-89714-807-8

Vandentorren, S., Bretin, P., Zeghnoun, A., Mandereau-Bruno, L., Croisier, A., Cochet, C., Ribéron, J., Siberan, I., Declercq, B., Ledrans, M., 2006. August 2003 heat wave in France: risk factors for death of elderly people living at home. Eur. J. Public Health, 16, 583-591. https://doi.org/10.1093/eurpub/ck1063

Verdonck M., Demuzere M., Bechtel B., Beck C., Brousse O., Droste A., Fenner D., Leconte F., Van Coillie F., 2019. The Human Influence Experiment (Part 2): Guidelines for improved mapping of Local Climate Zones using a supervised classification. Urban Science, 3, 27. https://doi.org/10.3390/urbansci3010027

Voogt J. A., Oke T. R., 2003. Thermal remote sensing of urban 
climates. Remote Sensing of Environment, Urban Remote Sensing, 86, 370-384. https://doi.org/10.1016/S00344257(03)00079-8

Weston K. J., 1987. Boundary layer climates (Second edition). By T. R. Oke, Methuen, Quarterly Journal of the Royal Meteorological
Society, 114, 435. https://doi.org/10.1002/qj.49711448412

Yu K., Chen Y., Wang D., Chen Z., Gong A., Li J., 2019. Study of the seasonal effect of building shadows on urban Land Surface Temperatures based on remote sensing data. Remote Sensing, 11, 497. https://doi.org/10.3390/rs11050497

Citation de l'article : Renard F. et Alonso L., 2020. Températures de surface et mesures mobiles confrontées aux zones climatiques locales : exemples des agglomérations de Tokyo et de Lyon. Climatologie, 17, 11. 